\title{
Spherical method for studying Wulff shapes and related topics
}

\author{
Huhe Han and Takashi Nishimura
}

\begin{abstract}
.
This is a survey article on the spherical method for studying Wulff shapes and related topics. The spherical method, which seems less common, is a powerful tool to study Wulff shapes and their related topics. It is verified how powerful the spherical method is by various results which seem difficult to be obtained without using the method. In this survey, the spherical method is explained in detail, and results obtained by using the spherical method until April 2016 are explained as well.
\end{abstract}

\section{Contents}

1. Introduction 2

2. Spherical method 9

2.1. Pedals 9

2.2. Spherical duals and spherical pedals $\quad 11$

$\begin{array}{ll}\text { 2.3. Spherical polar sets } & 15\end{array}$

2.4. Caustics and symmetry sets 18

2.5. Spherical Wulff shapes, spherical caustics and spherical symmetry sets

3. Several results obtained by using the spherical method 25

3.1. Crystallines 25

3.2. Aperture of plane curves 26

Received Month Day, Year.

Revised Month Day, Year.

2010 Mathematics Subject Classification. Primary 52A55; Secondary 52A20, 57R45, 82D25.

Key words and phrases. Wulff shape, Convex integrand, Pedal, Spherical method, Spherical dual, Spherical pedal, Spherical polar set, Spherical Wulff shape, Spherical caustic, Spherical symmetry set.

This work was partially supported by JSPS KAKENHI Grant Number 26610035 . 
3.3. The spherical polar transform 29

3.4. Convex integrands of class $C^{1} \quad 32$

3.5. Wulff shapes with $C^{1}$ boundary 35

3.6. Self-dual Wulff shapes and spherical Wulff shapes of constant width $\pi / 2 \quad 36$

3.7. Stability of $C^{\infty}$ convex integrands 37

3.8. Simultaneous stability of $C^{\infty}$ convex integrands and their duals $\quad 42$

4. Questions 45

4.1. On aperture of higher dimensional hypersurfaces 46

4.2. On the space consisting of convex integrands and the space consisting of Wulff shapes 48

4.3. On convex integrands of class $C^{k}(2 \leq k<\infty) \quad 49$

Acknowledgements $\quad 50$

\section{$\S 1 . \quad$ Introduction}

It is known that, in the last half of 19th century, J. W. Gibbs and P. Curie had already pointed out that the shape of a crystal at equilibrium is determined so that the total surface energy is minimized amongst all crystals having the same total volume of the given crystal (see for instance $[61,63])$. At the beginning of 20 th century, more precisely in 1901, in the epoch-making paper [73], G. Wulff gave an important formula, called Wulff's theorem later, for a crystalline (namely, the surface energy function for a crystal of polytope type) at equilibrium. This formula leads to a geometric method to construct the shape of crystal which satisfies Wulff's theorem, called Wulff's construction ${ }^{1}$, from the

\footnotetext{
${ }^{1}$ For the surface energy of a general crystal, it has a long history to show that "Wulff's construction gives the unique solution for the minimizing problem amongst all crystals having the same volume as the given crystal". The following is a quotation from [20].

A. Dinghas (1944, [14]) gave a formal proof. J. Taylor (1978, [69]) gave a precise proof for very general surface energies and a very general class of set for which the surface energy is defined by using geometric measure theory. B. Dacorogna and C. E. Pfister (1992, [13]) gave an analytic proof when $n=1$. I. Fonseca (1991, [16]) and I. Fonseca and S. Müller (1991, [17]) gave a simpler proof for arbitrary dimensions.
} 


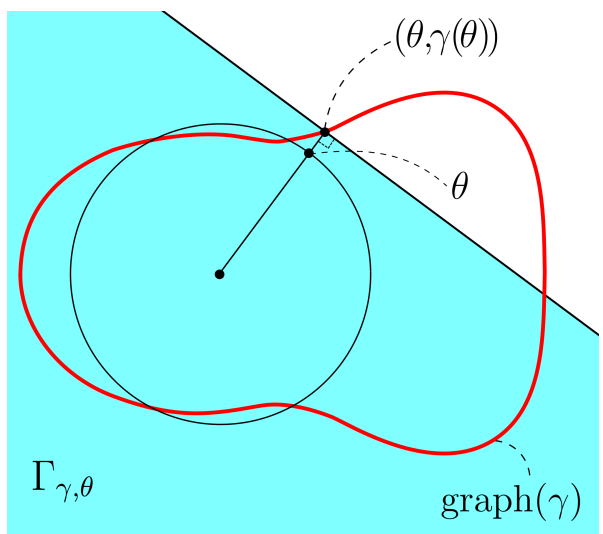

Fig. 1. The figure of $\Gamma_{\gamma, \theta}$.

known energy density $\gamma: S^{n} \rightarrow \mathbb{R}_{+}$. Nowadays, the shape of a crystal constructed by Wulff's construction is called the Wulff shape. Wulff shapes and their related topics are our main objects in this article. Our main tool to investigate them is the spherical method, which is a new and powerful tool. The main purpose of this article is to give a survey on "what the spherical method is" and "how powerful the spherical method is".

We start from explaining Wulff shapes. The most main character for studying Wulff shapes is a continuous function $\gamma: S^{n} \rightarrow \mathbb{R}_{+}$, where $n, S^{n}$ and $\mathbb{R}_{+}$are a positive integer, the unit sphere in $\mathbb{R}^{n+1}$ and the set consisting of positive real numbers respectively. Thus, first of all, we assume that a continuous function $\gamma: S^{n} \rightarrow \mathbb{R}_{+}$is given. For the given $\gamma$ and a point $\theta \in S^{n}$, the half space $\Gamma_{\gamma, \theta}$ is defined as follows, where $x \cdot \theta$ means the standard scalar product of two vectors $x, \theta$ of the vector space $\mathbb{R}^{n+1}$ (see Figure 1).

$$
\Gamma_{\gamma, \theta}=\left\{x \in \mathbb{R}^{n+1} \mid x \cdot \theta \leq \gamma(\theta)\right\} .
$$

Then, the Wulff shape associated with $\gamma$, denoted by $\mathcal{W}_{\gamma}$, is constructed as follows (see Figure 2).

$$
\mathcal{W}_{\gamma}=\bigcap_{\theta \in S^{n}} \Gamma_{\gamma, \theta}
$$

By definition, it is clear that $\mathcal{W}_{\gamma}$ is compact, convex and the origin $\mathbf{0}$ of $\mathbb{R}^{n+1}$ is contained in $\mathcal{W}_{\gamma}$ as an interior point of $\mathcal{W}_{\gamma}$. The constructed 


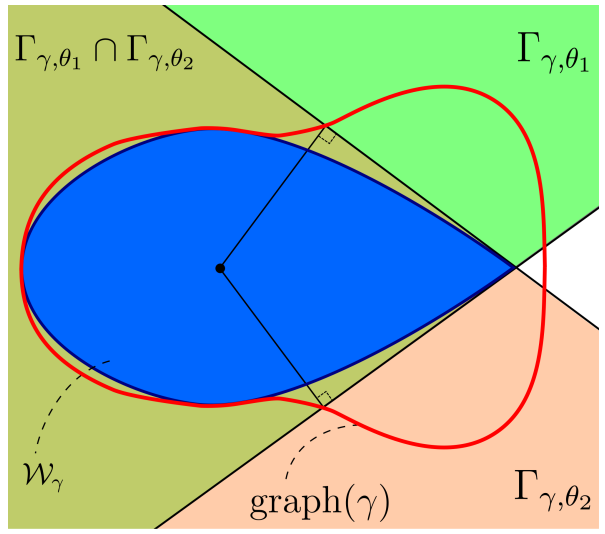

Fig. 2. The Wulff shape $\mathcal{W}_{\gamma}$ associated with $\gamma$.

shape $\mathcal{W}_{\gamma}$ is a geometric model of a crystal. Then, a natural question arises as follows:

Question 1. What does the given continuous function $\gamma$ mean physically?

Question 1 is answered as follows. Let $\partial \mathcal{W}_{\gamma}$ be the boundary of $\mathcal{W}_{\gamma}$. For any $\theta \in S^{n}$, the real number $\gamma(\theta)$ means the surface energy density of $\partial \mathcal{W}_{\gamma}$ at the direction $\theta \in S^{n}$, namely, at the point $\partial \mathcal{W}_{\gamma} \cap L_{\theta}$ where $L_{\theta}$ is the half line $\left\{r \theta \in \mathbb{R}^{n+1} \mid r \in \mathbb{R}_{+}\right\}$.

Let $C^{0}\left(S^{n}, \mathbb{R}_{+}\right)$be the set consisting of continuous function $S^{n} \rightarrow$ $\mathbb{R}_{+}$. The set $C^{0}\left(S^{n}, \mathbb{R}_{+}\right)$is a metric space endowed with the uniformly convergence metric. Let $\mathcal{H}_{c o n v, \mathbf{0}}\left(\mathbb{R}^{n+1}\right)$ be the set consisting of compact and convex subsets of $\mathbb{R}^{n+1}$ containing the origin $\mathbf{0}$ as an interior point. Let $\mathcal{H}\left(\mathbb{R}^{n+1}\right)$ be the set consisting of non-empty compact sets of $\mathbb{R}^{n+1}$. It is known that $\mathcal{H}\left(\mathbb{R}^{n+1}\right)$ is a complete metric space by the Pompeiu-Hausdorff metric (see for instance $[6,15]$ ). The set $\mathcal{H}_{c o n v, \mathbf{0}}\left(\mathbb{R}^{n+1}\right)$ is a subspace of $\mathcal{H}\left(\mathbb{R}^{n+1}\right)$. Notice that $\mathcal{H}_{\text {conv }, \mathbf{0}}\left(\mathbb{R}^{n+1}\right)$ is not complete. Then, by the Wulff construction, the following mapping defined by $\mathcal{W}(\gamma)=\mathcal{W}_{\gamma}$ is well-defined.

$$
\mathcal{W}: C^{0}\left(S^{n}, \mathbb{R}_{+}\right) \rightarrow \mathcal{H}_{c o n v, \mathbf{0}}\left(\mathbb{R}^{n+1}\right) .
$$

Although it is almost clear that the well-defined mapping $\mathcal{W}$ is continuous under the above topologies, the following fundamental questions on $\mathcal{W}$ naturally arise. 


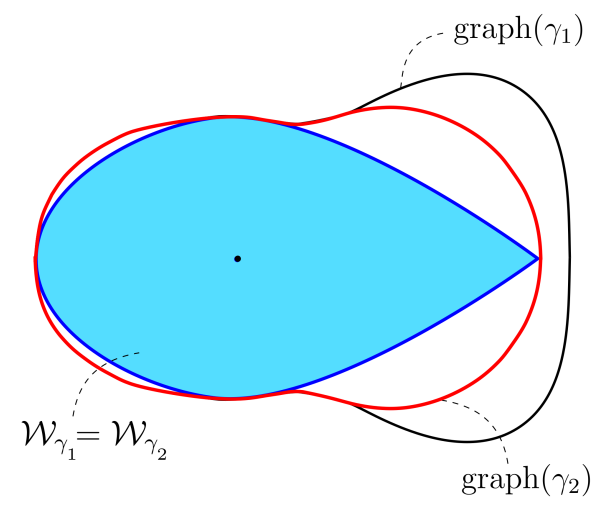

Fig. 3. A counterexample for Question 3.

Question 2. Is $\mathcal{W}$ surjective?

Question 3. Is $\mathcal{W}$ injective?

The affirmative answer to Question 2 can be found in [69]. Namely, for any $W \in \mathcal{H}_{\text {conv }, \mathbf{0}}\left(\mathbb{R}^{n+1}\right)$, it is known that $\mathcal{W}^{-1}(W) \neq \emptyset$. On the other hand, the answer to Question 3 is negative in general as follows. For any $\gamma \in C^{0}\left(S^{n}, \mathbb{R}_{+}\right)$, set

$$
\operatorname{graph}(\gamma)=\left\{(\theta, \gamma(\theta)) \in \mathbb{R}^{n+1}-\{\mathbf{0}\} \mid \theta \in S^{n}\right\},
$$

where $(\theta, \gamma(\theta))$ is the polar plot expression for a point of $\mathbb{R}^{n+1}-\{\mathbf{0}\}$. The mapping inv $: \mathbb{R}^{n+1}-\{\mathbf{0}\} \rightarrow \mathbb{R}^{n+1}-\{\mathbf{0}\}$ defined as follows is called the inversion with respect to the origin of $\mathbb{R}^{n+1}$.

$$
\operatorname{inv}(\theta, r)=\left(-\theta, \frac{1}{r}\right) .
$$

Let $\Gamma_{\gamma}$ be the boundary of the convex hull of $\operatorname{inv}(\operatorname{graph}(\gamma))$. Then, the following is known.

Proposition $1([69,23])$. Let $\gamma_{1}, \gamma_{2}$ be two elements of $C^{0}\left(S^{n}, \mathbb{R}_{+}\right)$ such that $\Gamma_{\gamma_{1}}=\Gamma_{\gamma_{2}}$. Then, the equality $\mathcal{W}_{\gamma_{1}}=\mathcal{W}_{\gamma_{2}}$ holds.

By Proposition 1, counterexamples for Question 3 are easily constructed (see Figure 3).

As the next question, the following question naturally arises. 


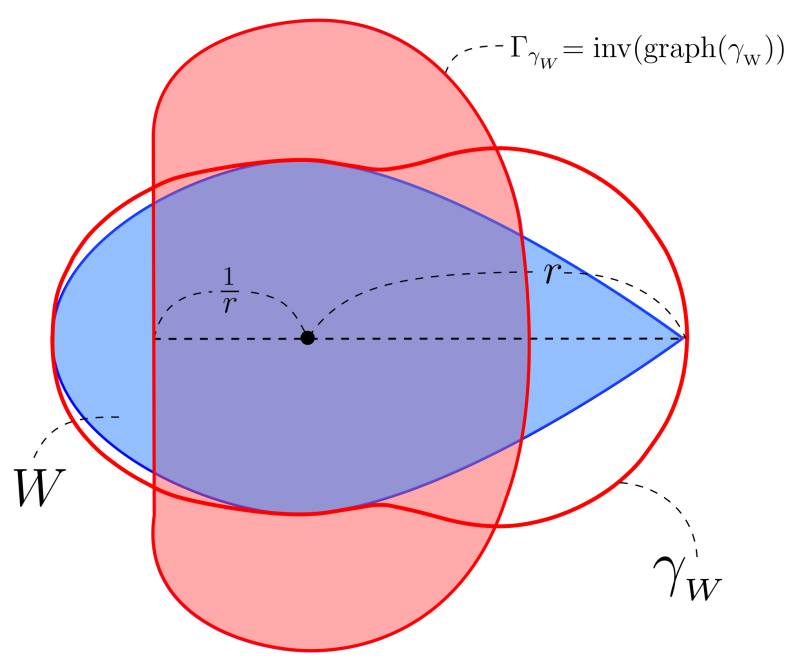

Fig. 4. The convex integrand $\gamma_{W}$ of $W$.

Question 4. Let $W$ be an element of $\mathcal{H}_{\text {conv }, 0}\left(\mathbb{R}^{n+1}\right)$, Then, characterize the best efficient element of $\mathcal{W}^{-1}(W)$ if it exists.

There is the well-known notion which may be considered as the ultimate answer to Question 4. Proposition 1 may be a clue to reach the following notion.

Definition 1. let $W$ be an element of $\mathcal{H}_{\text {conv }, \mathbf{0}}\left(\mathbb{R}^{n+1}\right)$. Let $\gamma_{W}$ : $S^{n} \rightarrow \mathbb{R}_{+}$be the continuous function satisfying inv $\left(\operatorname{graph}\left(\gamma_{W}\right)\right)=\Gamma_{\gamma_{W}}$. Then, the function $\gamma_{W}$ is called the convex integrand of $W$ (see Figure 4).

The notion of convex integrand was firstly introduced by J. Taylor in [69] and it plays a key role for studying Wulff shapes (for details on convex integrands, see for instance $[51,69])$. For any Wulff shape $W$ and any $\gamma \in \mathcal{W}^{-1}(W)$, since $\gamma(\theta)$ means the surface energy density of $\partial W$ at the direction $\theta \in S^{n}$, integrating the convex integrand $\gamma$ over $S^{n}$ represents the surface energy of $W$. Hence, $\gamma$ is called an integrand of $W$. And moreover, since $\operatorname{inv}\left(\operatorname{graph}\left(\gamma_{W}\right)\right)$ is exactly the boundary of a convex set, $\gamma_{W}$ is called the convex integrand of $W$.

Proposition 2. $[69,23]$ Let $W$ be an element of $\mathcal{H}_{\text {conv }, \mathbf{0}}\left(\mathbb{R}^{n+1}\right)$. Then, for any $\gamma \in \mathcal{W}^{-1}(W)$ and any $\theta \in S^{n}$, the following inequality 
holds.

$$
\gamma_{W}(\theta) \leq \gamma(\theta) .
$$

By Proposition 2, the convex integrand $\gamma_{W}$ may be regarded as the best efficient continuous function in $\mathcal{W}^{-1}(W)$.

Another question which can naturally and easily arises is the following.

Question 5. Characterize the element $W \in \mathcal{H}_{\text {conv }, \mathbf{0}}\left(\mathbb{R}^{n+1}\right)$ such that $\mathcal{W}^{-1}(W)$ consists of only the convex integrand of $W$.

In Subsection 3.5, a partial answer to Question 5 is given with its proof.

In order to understand the deep relation between a Wulff shape $W$ and its convex integrand $\gamma_{W}$ well, we introduce the notion of dual convex integrand for a given convex integrand. Given a convex integrand $\gamma: S^{n} \rightarrow \mathbb{R}_{+}$, define the continuous function $\delta: S^{n} \rightarrow \mathbb{R}_{+}$by the following equality, where $\partial \mathcal{W}_{\gamma}$ denotes the boundary of $\mathcal{W}_{\gamma}$.

$$
\operatorname{inv}(\operatorname{graph}(\delta))=\partial \mathcal{W}_{\gamma} .
$$

The defined function $\delta: S^{n} \rightarrow \mathbb{R}_{+}$is clearly a convex integrand, and it is called the dual convex integrand or dual of $\gamma$. The dual Wulff shape of $\mathcal{W}_{\gamma}$, denoted by $\mathcal{D} \mathcal{W}_{\gamma}$, is the Wulff shape associated with $\delta$.

$$
\mathcal{D} \mathcal{W}_{\gamma}=\mathcal{W}_{\delta}
$$

Concerning convex integrands and duals, the following question seems to be the most natural.

Question 6. (1) Is the dual convex integrand of $\delta \gamma$ ?

(2) Does the following involutive property hold?

$$
\mathcal{D D} \mathcal{W}_{\gamma}=\mathcal{W}_{\gamma} .
$$

The spherical method solves Question 6 affirmatively. See Proposition 8 in Subsection 2.5.

The next natural question on convex integrands and their duals is the following.

Question 7. Characterize the convex integrand $\gamma$ such that $\gamma=\delta$ in terms of the Wulff shape $\mathcal{W}_{\gamma}$.

Question 7 is equivalent to say that "Characterize the Wulff shape $\mathcal{W}_{\gamma}$ satisfying $\mathcal{W}_{\gamma}=\mathcal{D} \mathcal{W}_{\gamma}$ in terms of $\mathcal{W}_{\gamma}$, where $\gamma: S^{n} \rightarrow \mathbb{R}_{+}$is a given convex integrand". Thus, Question 7 is the characterization question of a self-dual Wulff shape. A complete answer to this question with a rigorous 
proof and with many examples, which is summarized in Subsection 3.6, is obtained in [24].

The following Question 8, which is also natural, is a characterization question on differentiability of a convex integrand.

Question 8. Let $k$ be a positive integer or $\infty$. Then, characterize a $C^{k}$ convex integrand $\gamma_{W}$ in terms of the Wulff shape $W$.

In the case $k=1$, a partial answer to Question 8 can be found in [51]. In [23], a complete answer to this question with a rigorous proof is obtained, and it is summarized in Subsection 3.4.

In the case $1<k<\infty$, as of May, 2016, it seems that Question 8 is still open. In Section 4, Question 8 is explained in detail with its history.

In the case $k=\infty$, independent partial answers to Question 8 can be found in $[1,51,68]$. In [8], a complete answer to this question, which is summarized in Subsection 3.8, is obtained.

In the case that a given convex integrand $\gamma: S^{n} \rightarrow \mathbb{R}_{+}$is of class $C^{\infty}$, the notion of stability is naturally defined. Let $C^{\infty}\left(S^{n}, \mathbb{R}_{+}\right)$be the set consisting of $C^{\infty}$ functions $S^{n} \rightarrow \mathbb{R}_{+}$. For the set $C^{\infty}\left(S^{n}, \mathbb{R}_{+}\right)$, there exists the natural topology, called Whitney $C^{\infty}$ topology. For details on Whitney $C^{\infty}$ topology see Subsection 3.7. Notice that $C^{\infty}\left(S^{n}, \mathbb{R}_{+}\right)$ may be regarded as a topological subspace of the topological space $C^{0}\left(S^{n}, \mathbb{R}_{+}\right)$. However, since any element of $C^{\infty}\left(S^{n}, \mathbb{R}_{+}\right)$is differentiable as many times as we want and we would like to investigate the stability of $C^{\infty}$ convex integrand, we prefer to regard $C^{\infty}\left(S^{n}, \mathbb{R}_{+}\right)$as the topological space endowed with Whitney $C^{\infty}$ topology. Let $S^{\infty}\left(S^{n}, \mathbb{R}_{+}\right)$be the subspace of $C^{\infty}\left(S^{n}, \mathbb{R}_{+}\right)$consisting of stable functions $S^{n} \rightarrow \mathbb{R}_{+}$. For details on stable functions also, see Subsection 3.7. Let $C_{\text {conv }}^{\infty}\left(S^{n}, \mathbb{R}_{+}\right)$ be the subspace of $C^{\infty}\left(S^{n}, \mathbb{R}_{+}\right)$consisting of $C^{\infty}$ convex integrand. By Mather's profound works on stability of $C^{\infty}$ mappings ([40, 41, 42, 43, $44,45])$, it is well-known that $S^{\infty}\left(S^{n}, \mathbb{R}_{+}\right)$is dense in $C^{\infty}\left(S^{n}, \mathbb{R}_{+}\right)$. However, this well-known fact is useless to answer the following question.

Question 9. Is the following intersection dense in $C_{\text {conv }}^{\infty}\left(S^{n}, \mathbb{R}_{+}\right)$?

$$
S^{\infty}\left(S^{n}, \mathbb{R}_{+}\right) \cap C_{\text {conv }}^{\infty}\left(S^{n}, \mathbb{R}_{+}\right) .
$$

Question 9 may be considered as the density question of stable functions in a restricted function space. Researches arisen from similar motivations as Question 9 can be found in [46, 12, 26, 27, 28, 29, 30]. Thus, from the viewpoint of Singularity Theory, Question 9 may be regarded as a natural question. A complete answer to Question 9, which is summarized in Subsection 3.7, can be found in [7]. 
The following Question 10, which is also natural, is the characterization question on stability of a $C^{\infty}$ convex integrand.

Question 10. Characterize a stable convex integrand $\gamma$ in terms of its dual convex integrand $\delta$.

In [8], a complete answer to Question 10, which is summarized in Subsection 3.8, is given. The answer given in [8] has the form that a $C^{\infty}$ convex integrand is stable if and only if its dual convex integrand $\delta$ is stable. By this answer to Question 10, the following question naturally arises.

Question 11. Let $\gamma: S^{n} \rightarrow \mathbb{R}_{+}$be a stable convex integrand.

(1) Suppose that $\theta_{0}$ is a non-degenerate critical point of $\gamma$. Then, is $-\theta_{0}$ a non-degenerate critical ponit of $\delta$ ?

(2) If the answer to (1) is affirmative, then are there some relations between the Morse index of $\gamma$ at $\theta_{0}$ and the Morse index of $\delta$ at $-\theta_{0}$ ?

A complete answer to Question 11, too, can be found in [8]. Summary of this result, too, is given in Subsection 3.8.

For the topics on Wulff shapes which are not treated in this survey article, refer to $[20,51,61,63,69,70]$.

This article is organized as follows. In Section 2, what is the spherical method is explained. In Section 3, our results obtained by using the spherical method until April of 2016 are gathered together. By Section 3 , it is expected that the readers can understand how powerful the spherical method is. Finally, in Section 4, it is posed several questions which are related to the topics of this article and seem to be open.

\section{$\S 2$. Spherical method}

\subsection{Pedals}

In Physics, pedals seem to be one of common backgrounds to study crystals (see for instance, [61, 63]). From our viewpoint as well, the notion of pedal for a smooth hypersurface in $\mathbb{R}^{n+1}$ is very important. Therefore, firstly in Section 2, pedals are quickly explained. As for the references of pedals, the authors recommend $[2,4,10]$ as excellent references for pedals from mathematical side.

Definition 2. Let $\Phi: S^{n} \rightarrow \mathbb{R}^{n+1}-\{\mathbf{0}\}$ be a $C^{1}$ embedding. Then, the pedal relative to the pedal point $\mathbf{0}$ for $\Phi$, denoted by $p d_{\Phi, \mathbf{0}}: S^{n} \rightarrow$ $\mathbb{R}^{n+1}$, is the mapping which maps $\theta \in S^{n}$ to the unique nearest point of 


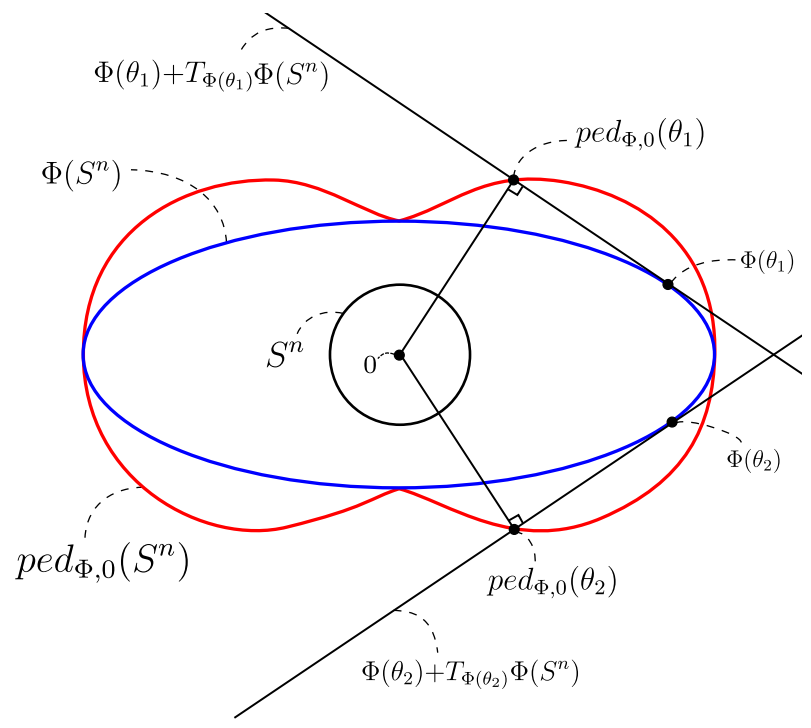

Fig. 5. The pedal relative to the pedal point $\mathbf{0}$ for $\Phi$.

$\Phi(\theta)+T_{\Phi(\theta)} \Phi\left(S^{n}\right)$ from the origin 0, where $\Phi(\theta)+T_{\Phi(\theta)} \Phi\left(S^{n}\right)$ stands for the affine tangent hyperplane to $\Phi\left(S^{n}\right)$ at $\Phi(\theta)$ (see Figure 5).

Why is the notion of pedal important? Let $\gamma: S^{n} \rightarrow \mathbb{R}_{+}$be a convex integrand. Suppose that the boundary of a Wulff shape $\partial \mathcal{W}_{\gamma}$ is the image of a $C^{\infty}$ embedding $\Phi: S^{n} \rightarrow \mathbb{R}^{n+1}-\{\mathbf{0}\}$. Then, by definition, the graph of the given convex integrand $\gamma: S^{n} \rightarrow \mathbb{R}^{n+1}-\{\mathbf{0}\}$ may be considered as the pedal relative to the pedal point $\mathbf{0}$ for $\Phi$. By using the spherical method, it is proved that $\gamma$ is of class $C^{\infty}$ (see Subsection 3.8). Since $\operatorname{graph}(\gamma)$ does not contain the origin, from the information of $\operatorname{ped}_{\Phi, \mathbf{0}}$ : $S^{n} \rightarrow \mathbb{R}^{n+1}$, the family of affine tangent hyperplanes to $\Phi\left(S^{n}\right)$ can be uniquely restored. In other words, ped $d_{\Phi, 0}: S^{n} \rightarrow \mathbb{R}^{n+1}$ is a method to store the family of affine tangent hyperplanes to $\Phi\left(S^{n}\right)$. In this sense, $\operatorname{ped}_{\Phi, \mathbf{0}}: S^{n} \rightarrow \mathbb{R}^{n+1}$ itself may be considered as a sort of Legendre transform for the hypersurface $\Phi\left(S^{n}\right)$. Since $\operatorname{ped}_{\Phi, \mathbf{0}}(\theta)$ can be expressed as $(\theta, \gamma(\theta))$ by using the polar plot expression and $\operatorname{inv}(\operatorname{graph}(\delta))=\partial \mathcal{W}_{\gamma}$ where $\delta$ is the dual convex integrand of $\gamma$, it follows that $\gamma$ may be regarded as the very Legendre transform of $\widehat{\delta}$ where $\hat{\delta}: S^{n} \rightarrow \mathbb{R}_{+}$is defined by

$$
\hat{\delta}(\theta)=\frac{1}{\delta(-\theta)} \quad\left(\forall \theta \in S^{n}\right) .
$$


And, in Physics, it is well-known Legendre transform is a very important notion for studying Wulff shapes (for instance, see pp.46-50 of [63]). Moreover, by using the spherical method, it can be proved out that $\mathcal{W}_{\gamma}$ is strictly convex if and only if the convex integrand $\gamma$ is of class $C^{1}$ (see Theorem 8 in Subsection 3.4). Thus, in our situation, if both $\gamma$ and $\delta$ are of class $C^{\infty}$, then both of $\mathcal{W}_{\gamma}$ and $\mathcal{W}_{\delta}$ are strictly convex. Therefore, we can expect that Wulff shapes with $C^{\infty}$ boundary are very nice objects so that the Legendre transform works very well. By these observations, one can understand why the notion of pedal is one of common backgrounds in physics.

Observe that both of Wulff shapes and pedals are defined by using perpendicular properties. Therefore, the unit sphere $S^{n+1}$ in $\mathbb{R}^{n+2}$ seems to be more suitable than $\mathbb{R}^{n+1}$ as the space where perpendicular properties are considered. This observation leads us to adopt the spherical method which deals with spherical counterparts of Wulff shapes, pedals etc. inside $S^{n+1}$ rather than inside $\mathbb{R}^{n+1}$.

\subsection{Spherical duals and spherical pedals}

Given a $C^{\infty}$ embedding $\Phi: S^{n} \rightarrow \mathbb{R}^{n+1}$, a $C^{\infty}$ embedding $\widetilde{\Phi}: S^{n} \rightarrow$ $S^{n+1}$ is firstly constructed, where $S^{n+1}$ is the unit sphere in $\mathbb{R}^{n+2}$. The north pole of $S^{n+1}$ is denoted by $N$, namely,

$$
N=(0, \ldots, 0,1) \in S^{n+1} \subset \mathbb{R}^{n+2} .
$$

The northern hemisphere of $S^{n+1}$ is denoted by $S_{N,+}^{n+1}$, namely,

$$
S_{N,+}^{n+1}=\left\{P \in S^{n+1} \mid N \cdot P>0\right\}
$$

where $N \cdot P$ stands for the standard scalar product of $(n+2)$-dimensional two vectors $N, P \in \mathbb{R}^{n+2}$. The mapping

$$
\mathbb{R}^{n+1} \ni x \mapsto(x, 1) \in \mathbb{R}^{n+1} \times\{1\} \subset \mathbb{R}^{n+2}
$$

is denoted by $I d$, namely,

$$
\operatorname{Id}(x)=(x, 1)
$$

The central projection with respect to $N$ is the mapping $\alpha_{N}: S_{N,+}^{n+1} \rightarrow$ $\mathbb{R}^{n+1} \times\{1\} \subset \mathbb{R}^{n+2}$ defined by

$$
\alpha_{N}\left(P_{1}, \ldots, P_{n+1}, P_{n+2}\right)=\left(\frac{P_{1}}{P_{n+2}}, \ldots, \frac{P_{n+1}}{P_{n+2}}, 1\right),
$$

where $P=\left(P_{1}, \ldots, P_{n+1}, P_{n+2}\right)$ is a point of $S_{N,+}^{n+1}$ (see Figure 6$)$. Then, 


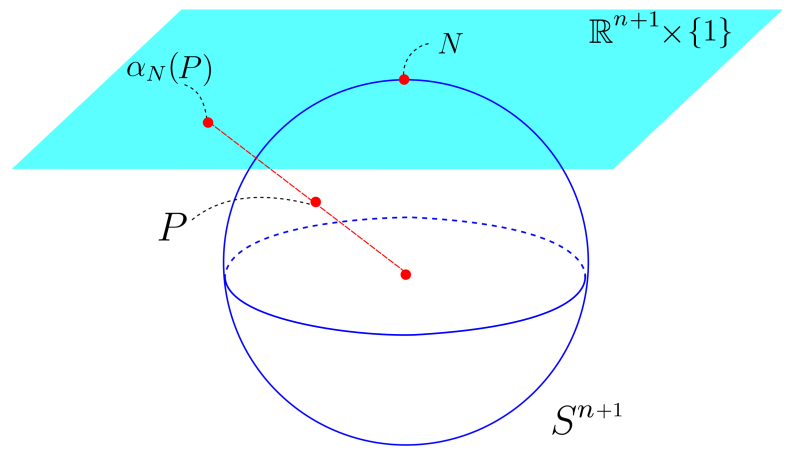

Fig. 6. The central projection with respect to $N$.

the mapping $\widetilde{\Phi}: S^{n} \rightarrow S_{N,+}^{n+1} \subset S^{n+1}$ derived from the given $C^{\infty}$ embedding $\Phi$ is defined as follows.

$$
\widetilde{\Phi}=\alpha_{N}^{-1} \circ I d \circ \Phi: S^{n} \rightarrow S_{N,+}^{n+1} \subset S^{n+1} .
$$

Definition 3. Let $\Phi: S^{n} \rightarrow \mathbb{R}^{n+1}$ be a $C^{\infty}$ embedding. Let $\widetilde{\Phi}$ : $S^{n} \rightarrow S_{N,+}^{n+1}$ be the $C^{\infty}$ embedding defined by $\widetilde{\Phi}=\alpha_{N}^{-1} \circ I d \circ \Phi$. Then, the mapping which maps $\theta \in S^{n}$ to the unique nearest point of $G H_{\widetilde{\Phi}(\theta)} \widetilde{\Phi}\left(S^{n}\right)$ from the north pole $N$ is denoted by s-ped $d_{\widetilde{\Phi}, N}: S^{n} \rightarrow S^{n+1}$ and is called the spherical pedal relative to the pedal point $N$ for $\widetilde{\Phi}$ (see Figure 7 ). Here, $G H_{\widetilde{\Phi}(\theta)} \widetilde{\Phi}\left(S^{n}\right)$ means the great hypersphere which is tangent to $\widetilde{\Phi}\left(S^{n}\right)$ at $\widetilde{\Phi}(\theta)$.

The spherical pedal relative to $N$ for $\widetilde{\Phi}$ is known to be decomposed into two simple mappings. In order to explain this decomposition, the spherical dual $D \widetilde{\Phi}: S^{n} \rightarrow S^{n+1}$ of $\widetilde{\Phi}$ is necessary.

Definition 4. The mapping $D \widetilde{\Phi}: S^{n} \rightarrow S^{n+1}$, called the spherical dual of $\widetilde{\Phi}$, is defined as the mapping which maps a point $\theta \in S^{n}$ to the point in $S_{N,+}^{n+1}$ such that $D \widetilde{\Phi}(\theta)$ is perpendicular to any $P \in$ $G H_{\widetilde{\Phi}(\theta)} \widetilde{\Phi}\left(S^{n}\right)$ (see Figure 8). 


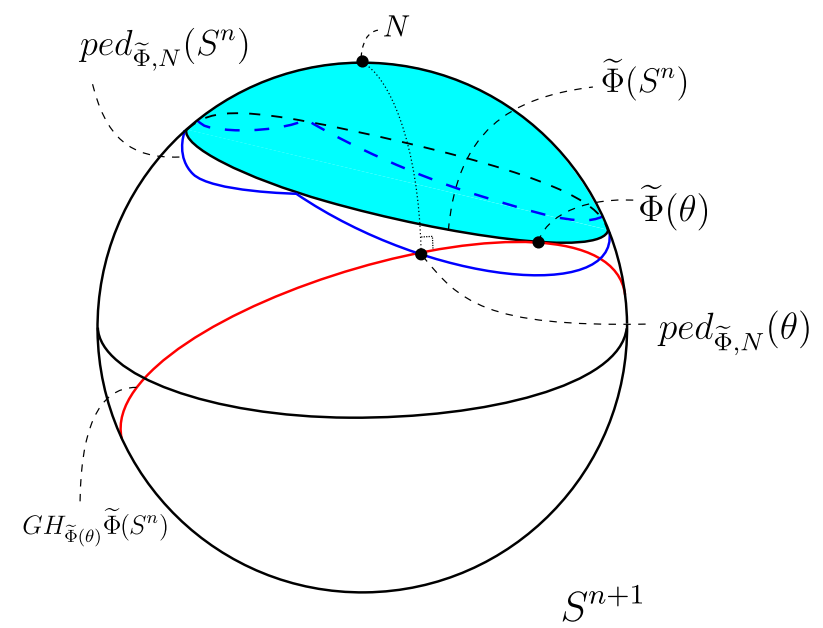

Fig. 7. The spherical pedal relative to $N$ for $\widetilde{\Phi}$.

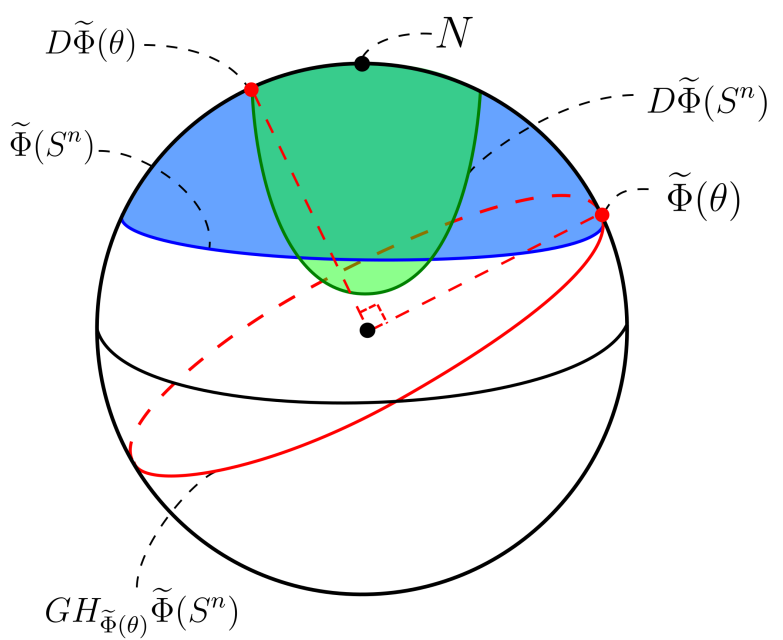

Fig. 8. The spherical dual of $\widetilde{\Phi}$.

It was V. I. Arnol'd who firstly defined the notion of spherical dual (for a spherical curve) (see [3]). Definition 4 is a natural generalization of his notion to spherical hypersurfaces. 


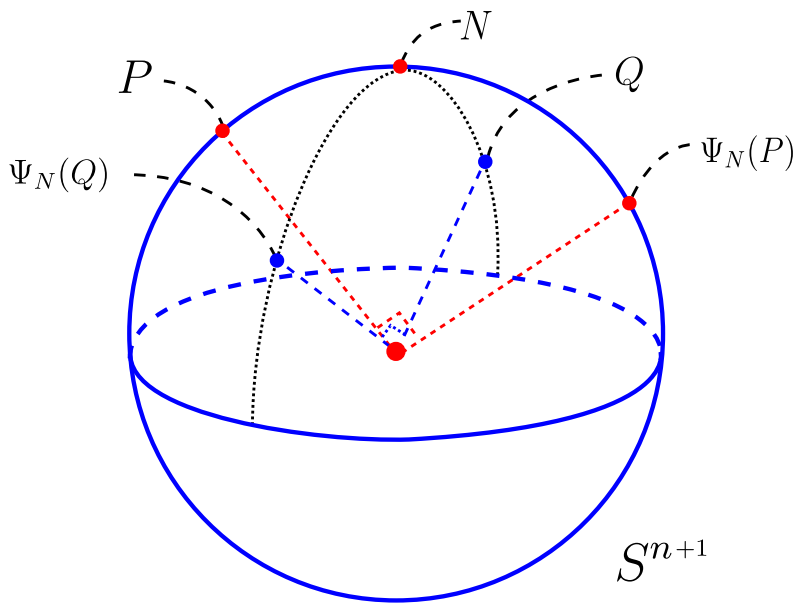

Fig. 9. The spherical blow-up relative to $N$. by

Next, we consider the mapping $\Psi_{N}: S^{n+1}-\{ \pm N\} \rightarrow S^{n+1}$ defined

$$
\Psi_{N}(P)=\frac{1}{\sqrt{1-(N \cdot P)^{2}}}(N-(N \cdot P) P)
$$

and depicted in Figure 9. By using $D \widetilde{\Phi}$ and $\Psi_{N}, s-p e d_{\widetilde{\Phi}, N}$ can be decomposed as follows.

Proposition 3 ([54]). s-ped $d_{\widetilde{\Phi}, N}=\Psi_{N} \circ D \widetilde{\Phi}$.

Notice that Proposition 3 has been proved only for spherical pedal curves in [54]. However, by the same proof, even when $n$ is greater than 1, s-ped $d_{\widetilde{\Phi}, N}$ can be decomposed as in Proposition 3. The mapping $\Psi_{N}$ has the following nice properties.

(1) For any $P \in S^{n+1}-\{ \pm N\}$, the equality $P \cdot \Psi_{N}(P)=0$ holds,

(2) for any $P \in S^{n+1}-\{ \pm N\}$, the property $\Psi_{N}(P) \in \mathbb{R} N+\mathbb{R} P$ holds,

(3) for any $P \in S^{n+1}-\{ \pm N\}$, the property $N \cdot \Psi_{N}(P)>0$ holds,

(4) the restriction $\left.\Psi_{N}\right|_{S_{N,+}^{n+1}-\{N\}}: S_{N,+}^{n+1}-\{N\} \rightarrow S_{N,+}^{n+1}-\{N\}$ is a $C^{\infty}$ diffeomorphism.

By these properties, it is reasonable to call the mapping $\Psi_{N}$ the spherical blow-up relative to $N$. The mapping $\Psi_{N}$ has been already used to study many topics related to perpendicularity. For instance, it was used for studying singularities of spherical pedal curves in $[36,53,54,55]$, for 
studying spherical pedal unfoldings in [56], for studying hedgehogs and no-silhouettes in [59], for studying (spherical) Wulff shapes in [60, 22, 23], and for studying the aperture of plane curves in [35]. A hyperbolic version of $\Psi_{N}$ is also useful in hyperbolic situation (see [34]). The above properties clearly derives the following:

Lemma $2.1([35])$. The inversion inv $: \mathbb{R}^{n+1}-\{\mathbf{0}\} \rightarrow \mathbb{R}^{n+1}-\{\mathbf{0}\}$ can be decomposed as follows.

$$
\operatorname{inv}=I d^{-1} \circ \alpha_{N} \circ \Psi_{N} \circ \alpha_{N}^{-1} \circ I d .
$$

In p. 50 of [2] and p. 91 of [4], the following assertion can be found.

Proposition $4([2,4])$. Let $\Phi: S^{n} \rightarrow \mathbb{R}^{n+1}-\{\mathbf{0}\}$ be a $C^{\infty}$ embedding. Then, the following equality holds.

$$
\operatorname{ped}_{\Phi, \mathbf{0}}=\operatorname{inv} \circ I d^{-1} \circ \alpha_{N} \circ D \widetilde{\Phi} .
$$

Here, $\widetilde{\Phi}=\alpha_{N}^{-1} \circ I d \circ \Phi$.

In the case that $\mathbf{0} \notin \Phi\left(S^{n}\right)$ and $\widetilde{\Phi}=\alpha_{N}^{-1} \circ I d \circ \Phi$, combining Lemma 2.1 and Proposition 4, one can easily obtain the proof of Proposition 3 as follows.

$$
\begin{aligned}
\operatorname{s-ped}_{\widetilde{\Phi}, N} & =\alpha_{N}^{-1} \circ I d \circ p e d_{\Phi, 0} \\
& =\alpha_{N}^{-1} \circ I d \circ \operatorname{inv} \circ I d^{-1} \circ \alpha_{N} \circ D \widetilde{\Phi} \\
& =\alpha_{N}^{-1} \circ I d \circ I d^{-1} \circ \alpha_{N} \circ \Psi_{N} \circ \alpha_{N}^{-1} \circ I d \circ I d^{-1} \circ \alpha_{N} \circ D \widetilde{\Phi} \\
& =\Psi_{N} \circ D \widetilde{\Phi} .
\end{aligned}
$$

However, in the case that $\mathbf{0} \in \Phi\left(S^{n}\right)$, it is impossible to apply Proposition 4. Moreover, in such a case, it seems impossible to investigate $\operatorname{ped}_{\Phi, \mathbf{0}}$ by the standard method. Nevertheless, via $\alpha_{N}^{-1} \circ I d$, we can investigate $p e d_{\Phi, \mathbf{0}}$ by using the spherical method. This is one of merits of the spherical method, and this plays one of the most important roles in Subsection 3.2.

\subsection{Spherical polar sets}

The notion of spherical polar set seems to be less common though it seems that the notion of polar set in $\mathbb{R}^{n+1}$ is relatively common (for instance, see [47]). Since the notion of spherical polar set is one of the major ingredients for the spherical method, in this subsection, properties of spherical polar sets in $S^{n+1}$ are quickly reviewed.

Given a point $P$ of $S^{n+1}, H(P)$ is the following set:

$$
H(P)=\left\{Q \in S^{n+1} \mid P \cdot Q \geq 0\right\} .
$$


Namely, $H(P)$ is the hemisphere with boundary centered at $P$.

Definition 5. For any subset $\widetilde{X}$ of $S^{n+1}$, the set $\widetilde{X}^{\circ}$ defined by

$$
\widetilde{X}^{\circ}=\bigcap_{P \in \widetilde{X}} H(P)
$$

is called the spherical polar set of $\widetilde{X}$.

It is clearly seen that for any $\widetilde{X} \subset S^{n+1}, \widetilde{X}^{\circ}$ is closed.

Lemma 2.2 ([60]). Given two subsets $\widetilde{X}, \widetilde{Y} \subset S^{n+1}$, we suppose that the inclusion $\widetilde{X} \subset \widetilde{Y}$ holds. Then, for the spherical polar sets $\widetilde{X}^{\circ}, \widetilde{Y}^{\circ}$, the inclusion $\widetilde{Y}^{\circ} \subset \widetilde{X}^{\circ}$ holds.

Lemma $2.3([60])$. Let $\widetilde{X}$ be a subset of $S^{n+1}$. Then, the inclusion $\widetilde{X} \subset \widetilde{X}^{\circ \circ}$ is satisfied.

Definition 6. For a subset $\widetilde{X} \subset S^{n+1}$, if there exists a point $P \in$ $S^{n+1}$ such that $H(P) \cap \widetilde{X}=\emptyset$, then $\widetilde{X}$ is said to be hemispherical.

For a hemispherical two-point set $\{P, Q\}$ of $S^{n+1}, P Q$ stands for the following arc:

$$
P Q=\left\{\frac{(1-t) P+t Q}{\|(1-t) P+t Q\|} \in S^{n+1} \mid 0 \leq t \leq 1\right\} .
$$

Notice that since $\{P, Q\}$ is hemispherical in the above, it follows that $\|(1-t) P+t Q\| \neq 0$ for any $t \in[0,1]$.

Definition 7. (1) Let $\widetilde{X}$ be a hemispherical subset of $S^{n+1}$. Then, $\widetilde{X}$ is said to be spherical convex if $P Q \subset \widetilde{X}$ for any $P, Q \in \widetilde{X}$.

(2) Let $\widetilde{X}$ be a hemispherical subset of $S^{n+1}$. Then, $\widetilde{X}$ is said to be strictly spherical convex if $P Q-\{P, Q\}$ is a subset of $\operatorname{int}(\widetilde{X})$ for any $P, Q \in \widetilde{X}$, where $\operatorname{int}(\widetilde{X})$ is the set consisting of interior points of $\widetilde{X}$.

(3) Let $\widetilde{X}$ be a hemispherical subset of $S^{n+1}$. Then, $\widetilde{X}$ is called a spherical convex body if $\widetilde{X}$ is closed and spherical convex and it has an interior point.

In general, $\widetilde{X}^{\circ}$ is not necessarily spherical convex even if $\widetilde{X}$ is hemispherical (for instance if $\widetilde{X}=\{P\}$ then $\widetilde{X}^{\circ}=H(P)$ is not spherical convex). However, it is easily seen that if $\widetilde{X}$ is hemispherical and has an interior point, then $\widetilde{X}^{\circ}$ is spherical convex. 
Lemma 2.4 ([60]). Let $\left\{\widetilde{X}_{\lambda} \subset S^{n+1}\right\}_{\lambda \in \Lambda}$ be a family of spherical convex subsets. Then, the intersection

$$
\bigcap_{\lambda \in \Lambda} \tilde{X}_{\lambda}
$$

is spherical convex as well.

Definition 8. Let $\widetilde{X}$ be a hemispherical subset of $S^{n+1}$. Define the set s-conv $(\widetilde{X})$ as follows.

$$
\mathrm{s}-\operatorname{conv}(\widetilde{X})=\left\{\frac{\sum_{i=1}^{k} t_{i} P_{i}}{\left\|\sum_{i=1}^{k} t_{i} P_{i}\right\|} \mid P_{i} \in \widetilde{X}, \sum_{i=1}^{k} t_{i}=1, t_{i} \geq 0, k \in \mathbb{N}\right\} .
$$

The set s-conv $(\widetilde{X})$ is called the spherical convex hull of $\widetilde{X}$.

It is clear that if $\widetilde{X}$ is spherical convex, then the equality s-conv $(\widetilde{X})=\tilde{X}$ holds. More generally, the following holds:

Lemma 2.5 ([60]). Let $\widetilde{X}$ be a hemispherical subset of $S^{n+1}$. Then, the spherical convex hull of $\widetilde{X}$ is the smallest spherical convex set containing $\widetilde{X}$, that is to say, the intersection of all spherical convex sets containing $\tilde{X}$.

Definition 9. Let $\left\{P_{1}, \ldots, P_{k}\right\}$ be a hemispherical finite subset of $S^{n+1}$ such that the spherical convex hull s-conv $\left(\left\{P_{1}, \ldots, P_{k}\right\}\right)$ has an interior point. Then, s-conv $\left(\left\{P_{1}, \ldots, P_{k}\right\}\right)$ is called the spherical polytope generated by $P_{1}, \ldots, P_{k}$.

Proposition $5([19,60])$. Let $\widetilde{X}$ be a closed hemispherical subset of $S^{n+1}$. Then, the following equality holds:

$$
\operatorname{s-conv}(\widetilde{X})=(\operatorname{s-conv}(\widetilde{X}))^{\circ \circ} .
$$

Notice the following:

(1) For any closed hemispherical subset $\widetilde{X} \subset S^{n+1}, \operatorname{s-conv}(\widetilde{X})$, too, is closed and hemispherical.

(2) For any subset $\widetilde{X} \subset S^{n+1}$, the inclusion $\widetilde{X} \subset \widetilde{X}^{\circ \circ}$ always holds by Lemma 2.3. On the other hand, even if $\widetilde{X}$ is closed and hemispherical, the inverse inclusion $\widetilde{X} \supset \widetilde{X}^{\circ \circ}$ is not satisfied in general.

Lemma $2.6([60])$. Let $\widetilde{X}=\left\{P_{1}, \ldots, P_{k}\right\} \subset S^{n+1}$ be a hemispherical finite subset of $S^{n+1}$. Then, the following equality holds:

$$
\left\{\frac{\sum_{i=1}^{k} t_{i} P_{i}}{\left\|\sum_{i=1}^{k} t_{i} P_{i}\right\|} \mid P_{i} \in \widetilde{X}, \sum_{i=1}^{k} t_{i}=1, t_{i} \geq 0\right\}^{\circ}=H\left(P_{1}\right) \cap \cdots \cap H\left(P_{k}\right) .
$$




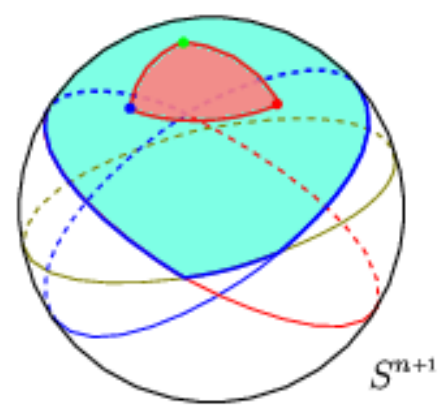

Fig. 10. Maehara's lemma.

Lemma 2.6 is depicted in Figure 10. The original form of Lemma 2.6 can be found in Maehara's book [39]. Thus, Lemma 2.6 is called Maehara's lemma.

\subsection{Caustics and symmetry sets}

When we want to study $C^{\infty}$ convex integrands, especially the stability of them, we notice that spherical caustics and spherical symmetry sets are powerful tools. Thus, before explaining spherical caustics and spherical symmetry sets, in this subsection we quickly review the caustic and the symmetry set for a $C^{\infty}$ embedding $S^{n} \rightarrow \mathbb{R}^{n+1}-\{\mathbf{0}\}$.

For any $C^{\infty}$ embedding $\Phi: S^{n} \rightarrow \mathbb{R}^{n+1}-\{\mathbf{0}\}$, the following family of functions $F: \mathbb{R}^{n+1} \times S^{n} \rightarrow \mathbb{R}$ is considered.

$$
F(v, \theta)=\frac{1}{2}\|\Phi(\theta)-v\|^{2} .
$$

It is easily seen that $F$ itself may be regarded as the mapping

$$
F: \mathbb{R}^{n+1} \rightarrow C^{\infty}\left(S^{n}, \mathbb{R}\right)
$$

which maps $v \in \mathbb{R}^{n+1}$ to $f_{v} \in C^{\infty}\left(S^{n}, \mathbb{R}\right)$, where $f_{v}$ is the mapping defined by

$$
f_{v}(\theta)=F(v, \theta) .
$$

Then, the caustic of $\Phi$, denoted by Caust $(\Phi)$ (or $\left.\operatorname{Caust}\left(\Phi\left(S^{n}\right)\right)\right)$, is the set consisting of vectors $v$ for which $f_{v}$ has a degenerate critical point (see Figure 11).

$$
\operatorname{Caust}(\Phi)=\left\{v \in \mathbb{R}^{n+1} \mid f_{v} \text { has a degenerate critical point }\right\} .
$$




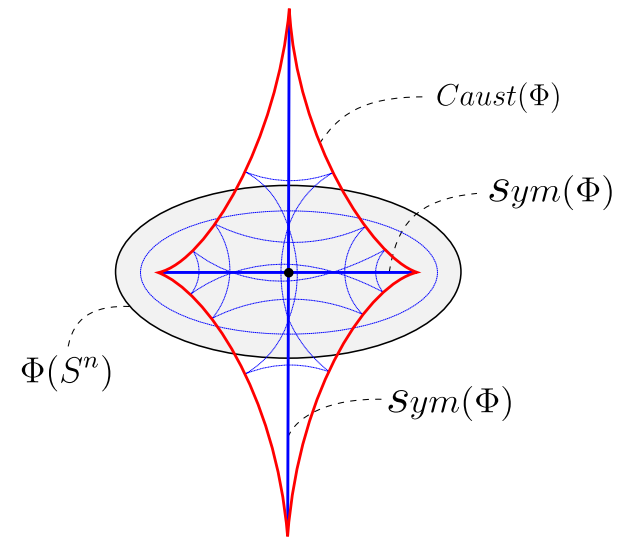

Fig. 11. The caustic and the symmetry set of $\Phi$.

The caustic of $\Phi$ has been well-investigated in Singularity Theory. For details on caustics, for example see $[2,4,5,31,32,33]$.

Next, for any $C^{\infty}$ embedding $\Phi: S^{n} \rightarrow \mathbb{R}^{n+1}-\{\mathbf{0}\}$, we review the symmetry set of $\Phi$. The symmetry set of $\Phi$ is defined by using the same mapping $f_{v}$ defined above. The set consisting of vectors $v$ for which $f_{v}$ has a multiple critical value is called the symmetry set of $\Phi$ and is denoted by $\operatorname{Sym}(\Phi)$ (or $\operatorname{Sym}\left(\Phi\left(S^{n}\right)\right)$ ) (see Figure 11).

$$
\operatorname{Sym}(\Phi)=\left\{v \in \mathbb{R}^{n+1} \mid f_{v} \text { has a multiple critical value }\right\}
$$

Although there are several literature on symmetry sets (for example $[9,10,11])$, it seems that, comparing with $\operatorname{Caust}(\Phi)$, symmetry sets have been less-investigated.

Given a $C^{\infty}$ embedding $\Phi: S^{n} \rightarrow \mathbb{R}^{n+1}-\{\mathbf{0}\}$, for any $t \in \mathbb{R}$, the $C^{\infty}$ mapping $\Phi_{t}: S^{n} \rightarrow \mathbb{R}^{n+1}$ is defined as follows. Given a $\theta \in S^{n}$, $L_{\Phi(\theta)}$ is defined as the line passing through $\Phi(\theta)$ which is perpendicular to $\Phi\left(S^{n}\right)$ at $\Phi(\theta)$. For any non-zero real number $t$, there exist exactly two points $P_{1}(\theta), P_{2}(\theta) \in L_{\Phi(\theta)}$ satisfying

$$
\left\|P_{1}(\theta)-\Phi(\theta)\right\|^{2}=\left\|P_{2}(\theta)-\Phi(\theta)\right\|^{2}=t^{2} .
$$

It is clear that, if $t$ is sufficiently near zero, then exactly one of $P_{1}(\theta)$ and $P_{2}(\theta)$ must be inside the connected region of $\mathbb{R}^{n+1}-\Phi\left(S^{n}\right)$ containing 0. Thus, without loss of generality, we may assume that $P_{1}(\theta)$ is inside 
the connected region. Then, for any non-zero real number $t$, define the mapping $\Phi_{t}: S^{n} \rightarrow \mathbb{R}^{n+1}$ by

$$
\Phi_{t}(\theta)=P_{1}(\theta)\left(\text { resp. }, \Phi_{t}(\theta)=P_{2}(\theta)\right)
$$

if $t$ is positive (resp., $t$ is negative). For $t=0$, define

$$
\Phi_{0}(\theta)=\Phi(\theta)
$$

The mapping $\Phi_{t}: S^{n} \rightarrow \mathbb{R}^{n+1}$ is called the wave front of $\Phi$. For any $t \in \mathbb{R}$, the wave front of $\Phi$ is clearly a $C^{\infty}$ mapping. It is easily seen that the caustic of $\Phi$ and the symmetry set of $\Phi$ can be characterized by the wave fronts of $\Phi$ as follows (see Figure 11).

Proposition 6.

$$
\operatorname{Caust}(\Phi)=\bigcup_{t \in \mathbb{R}} \Phi_{t}\left(S\left(\Phi_{t}\right)\right),
$$

where $S\left(\Phi_{t}\right)$ is the set consisting of singular points of $\Phi_{t}$.

(2)

$$
\operatorname{Sym}(\Phi)=\bigcup_{t \in \mathbb{R}}\left\{\Phi_{t}\left(\theta_{1}\right)=\Phi_{t}\left(\theta_{2}\right) \mid \theta_{1}, \theta_{2} \in S^{n}, \theta_{1} \neq \theta_{2}\right\} .
$$

In Subsection 3.7, the union

$$
\operatorname{Caust}(\Phi) \bigcup \operatorname{Sym}(\Phi)
$$

will play an important role.

\subsection{Spherical Wulff shapes, spherical caustics and spher- ical symmetry sets}

Definition 10. For any Wulff shape $\mathcal{W}_{\gamma}$, the spherical Wulff shape induced by $\mathcal{W}_{\gamma}$, denoted by $\widetilde{\mathcal{W}}_{\gamma}$, is the image of $\mathcal{W}_{\gamma}$ by $\alpha_{N}^{-1} \circ I d: \mathbb{R}^{n+1} \rightarrow$ $S_{N,+}^{n+1}$ (see Figure 13).

$$
\widetilde{\mathcal{W}}_{\gamma}=\alpha_{N}^{-1} \circ \operatorname{Id}\left(\mathcal{W}_{\gamma}\right)
$$

Any spherical Wulff shape $\widetilde{\mathcal{W}}_{\gamma}$ can be characterized by using the spherical blow-up $\Psi_{N}$ and the spherical polar set operation as follows:

$$
\widetilde{\mathcal{W}}_{\gamma}=\left(\Psi_{N} \circ \alpha_{N}^{-1} \circ \operatorname{Id}(\operatorname{graph}(\gamma))\right)^{\circ} .
$$




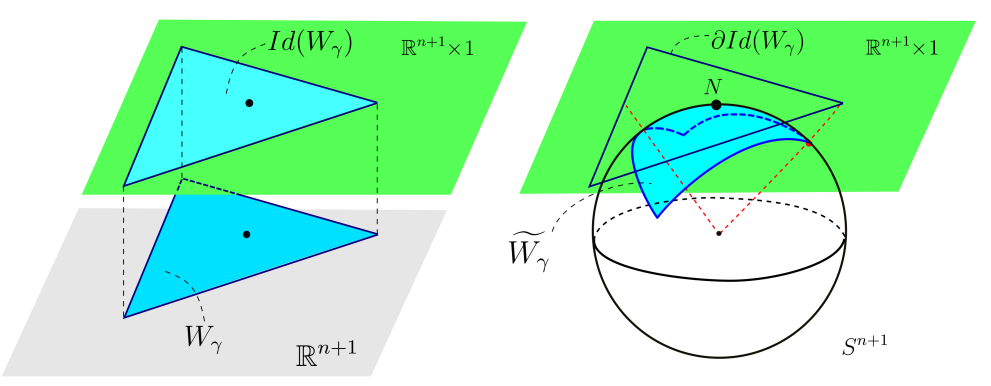

Fig. 12. The spherical Wulff shape associated with $\mathcal{W}_{\gamma}$.

Let $\widetilde{\mathcal{W}}_{\gamma}$ be a spherical Wulff shape. Then, the spherical dual of $\widetilde{\mathcal{W}}_{\gamma}$, denoted by $\mathcal{D} \widetilde{\mathcal{W}}_{\gamma}$, is the spherical polar set $\left(\widetilde{\mathcal{W}}_{\gamma}\right)^{\circ}$.

$$
\mathcal{D} \widetilde{\mathcal{W}}_{\gamma}=\left(\widetilde{\mathcal{W}}_{\gamma}\right)^{\circ}
$$

It is clear that any spherical Wulff shape $\mathcal{W}_{\gamma}$ is closed, hemispherical and spherical convex. Hence, by Proposition 5 we have the following.

\section{Proposition 7.}

$$
\mathcal{D} \mathcal{D} \widetilde{\mathcal{W}}_{\gamma}=\widetilde{\mathcal{W}}_{\gamma}
$$

By definition, it is clearly seen that $I d^{-1} \circ \alpha_{N}\left(\mathcal{D} \widetilde{\mathcal{W}}_{\gamma}\right)=\mathcal{D} \mathcal{W}_{\gamma}$. Hence, by Proposition 7 we have the assertion (1) of Proposition 8. The assertion (2) of Proposition 8 is a corollary of the assertion (1) of Proposition 8.

Proposition 8.

(1)

$$
\mathcal{D D} \mathcal{W}_{\gamma}=\mathcal{W}_{\gamma}
$$

(2) Suppose that $\gamma: S^{n} \rightarrow \mathbb{R}_{+}$is a convex integrand. Then, the dual convex integrand of the dual convex integrand of $\gamma$ is $\gamma$.

Next, suppose that $\widetilde{\mathcal{W}}_{\gamma}$ be a spherical Wulff shape such that the boundary of it is the image of a $C^{\infty}$ embedding $\widetilde{\Phi}: S^{n} \rightarrow S_{N,+}^{n+1}$. Then, we can define the Spherical Caustic and Spherical Symmetry Set for the embedding $\widetilde{\Phi}$ as follows. Let $d: S^{n+1} \times S^{n+1}-\triangle \rightarrow \mathbb{R}$ be the 
function such that $d\left(P_{1}, P_{2}\right)$ is the square of $P_{1} P_{2}$ where $\triangle=\{(P,-P) \in$ $\left.S^{n+1} \times S^{n+1} \mid P \in S^{n+1}\right\}$. We would like to consider the family of functions $\widetilde{F}: S_{N,+}^{n+1} \times S^{n} \rightarrow \mathbb{R}$ defined by

$$
\widetilde{F}(v, \theta)=\frac{1}{2} d(\widetilde{\Phi}(\theta), v)
$$

Then, $\widetilde{F}$ itself may be regarded as the mapping

$$
\widetilde{F}: S_{N,+}^{n+1} \rightarrow C^{\infty}\left(S^{n}, \mathbb{R}\right)
$$

which maps $v \in S_{N,+}^{n+1}$ to $\tilde{f}_{v} \in C^{\infty}\left(S^{n}, \mathbb{R}_{+}\right)$, where $\tilde{f}_{v}$ is the mapping defined by

$$
\widetilde{f}_{v}(\theta)=\widetilde{F}(v, \theta) .
$$

The Spherical Caustic of $\widetilde{\Phi}$, denoted by Spherical-Caust $(\widetilde{\Phi})$ (or Spherical$\left.\operatorname{Caust}\left(\widetilde{\Phi}\left(S^{n}\right)\right)\right)$, is the set consisting of vectors $v$ for which $\widetilde{f}_{v}(\theta)$ has a degenerate critical point form.

Spherical-Caust $(\widetilde{\Phi})=\left\{v \in S_{N,+}^{n+1} \mid \widetilde{f}_{v}\right.$ has a degenerate critical point $\}$.

The Spherical Symmetry Set of $\widetilde{\Phi}$, denoted by Spherical-Sym $(\widetilde{\Phi})$ (or Spherical-Sym $\left.\left(\widetilde{\Phi}\left(S^{n}\right)\right)\right)$, is the set consisting of vectors $v$ for which $\widetilde{f}_{v}(\theta)$ has a multiple critical value.

$$
\text { Spherical-Sym }(\widetilde{\Phi})=\left\{v \in S_{N,+}^{n+1} \mid \widetilde{f}_{v} \text { has a multiple critical value }\right\} .
$$

Given a $C^{\infty}$ embedding $\widetilde{\Phi}: S^{n} \rightarrow S_{N,+}^{n+1}-\{N\}$, for any $t \in \mathbb{R}(-\pi<$ $t<\pi$ ), the $C^{\infty}$ mapping $\widetilde{\Phi}_{t}: S^{n} \rightarrow S^{n+1}$ is defined as follows. Given a $\theta \in S^{n}, G C_{\widetilde{\Phi}(\theta)}$ is defined as the great circle passing through $\widetilde{\Phi}(\theta)$ which is perpendicular to $\widetilde{\Phi}\left(S^{n}\right)$ at $\widetilde{\Phi}(\theta)$. For any non-zero real number $t(-\pi<t<\pi)$, there exist exactly two points $P_{1}(\theta), P_{2}(\theta) \in G C_{\widetilde{\Phi}(\theta)}$ satisfying

$$
d\left(P_{1}(\theta), \widetilde{\Phi}(\theta)\right)=d\left(P_{2}(\theta), \widetilde{\Phi}(\theta)\right)=t^{2} .
$$

It is clear that, if $t$ is sufficiently near zero, then exactly one of $P_{1}(\theta)$ and $P_{2}(\theta)$ must be inside the connected region of $S^{n+1}-\widetilde{\Phi}\left(S^{n}\right)$ containing $N$. Thus, without loss of generality, we may assume that $P_{1}(\theta)$ is inside the connected region. Then, for any non-zero real number $t(-\pi<t<\pi)$, define the mapping $\widetilde{\Phi}_{t}: S^{n} \rightarrow S^{n+1}$ by

$$
\widetilde{\Phi}_{t}(\theta)=P_{1}(\theta)\left(\text { resp., } \widetilde{\Phi}_{t}(\theta)=P_{2}(\theta)\right)
$$



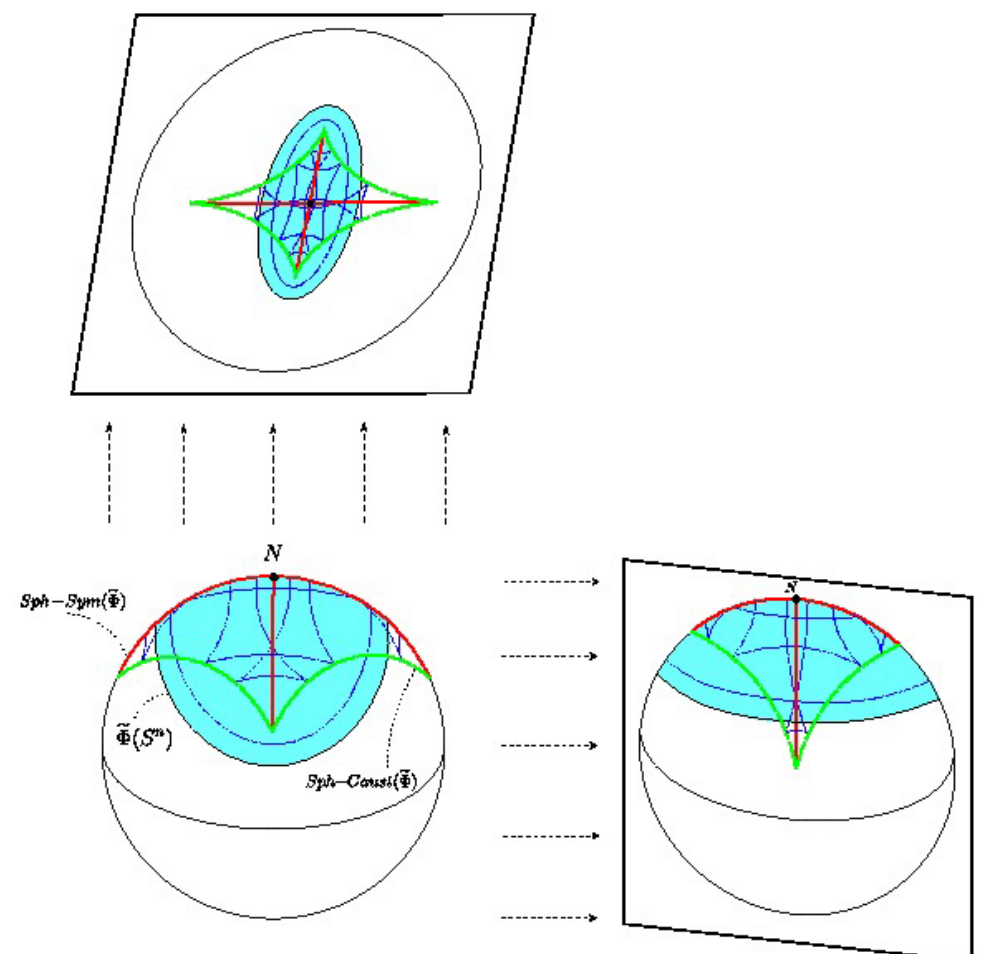

Fig. 13. The spherical caustic and the spherical symmetry set of $\widetilde{\Phi}$.

if $t$ is positive (resp., $t$ is negative). For $t=0$, define

$$
\widetilde{\Phi}_{0}(\theta)=\widetilde{\Phi}(\theta) .
$$

The mapping $\widetilde{\Phi}_{t}: S^{n} \rightarrow S^{n+1}$ is called the spherical wave front of $\widetilde{\Phi}$. For any $t \in \mathbb{R}$, the spherical wave front of $\widetilde{\Phi}$ is clearly a $C^{\infty}$ mapping. Similarly as the case of $C^{\infty}$ embedding $\Phi: S^{n} \rightarrow \mathbb{R}^{n+1}-\{\mathbf{0}\}$, it is easily seen that the spherical caustic of $\widetilde{\Phi}$ and the spherical symmetry set of $\widetilde{\Phi}$ can be characterized by the spherical wave fronts of $\widetilde{\Phi}$ as follows (see Figure 13). 
Proposition 9.

$$
\text { Spherical-Caust }(\widetilde{\Phi})=\bigcup_{-\pi<t<\pi} \widetilde{\Phi}_{t}\left(S\left(\widetilde{\Phi}_{t}\right)\right),
$$

$$
\text { where } S\left(\widetilde{\Phi}_{t}\right) \text { is the set consisting singular points of } \widetilde{\Phi}_{t} \text {. }
$$

Spherical-Sym $(\widetilde{\Phi})=\bigcup_{-\pi<t<\pi}\left\{\widetilde{\Phi}_{t}\left(\theta_{1}\right)=\widetilde{\Phi}_{t}\left(\theta_{2}\right) \mid \theta_{1}, \theta_{2} \in S^{n}, \theta_{1} \neq \theta_{2}\right\}$.

In the case of $\widetilde{\Phi}: S^{n} \rightarrow S_{N,+}^{n+1}-\{N\}$, we have more. It is clear that

$$
\widetilde{\Phi}_{\pi / 2}\left(S^{n}\right)=\partial \mathcal{D} \widetilde{\mathcal{W}}_{\gamma}
$$

By this fact and proposition 7, considering inside the sphere $S^{n+1}$ seems to derive a reasonable situation. Moreover, notice that, by [24], the selfdual Wulff shapes are strongly related to the angle $\pi / 2$ (see Subsection 3.6). Thus, the angle $\pi / 2$ may be considered as a significant number for studying Wulff shapes, although in $\mathbb{R}^{n+1}$ there are no such significant real numbers for studying Wulff shapes.

Definition 11. A Legendrian map-germ is a $C^{\infty}$ map-germ $f$ : $\left(\mathbb{R}^{n}, \mathbf{0}\right) \rightarrow\left(\mathbb{R}^{n+1}, \mathbf{0}\right)$ such that there exists a germ of $C^{\infty}$ vector field $\nu_{f}$ along $f$ satisfying the following two:

(1)

$$
\frac{\partial f}{\partial x_{1}}(x) \cdot \nu_{f}(x)=\cdots=\frac{\partial f}{\partial x_{n}}(x) \cdot \nu_{f}(x)=0 .
$$

(2) The map-germ $L_{f}:\left(\mathbb{R}^{n}, \mathbf{0}\right) \rightarrow T_{1} \mathbb{R}^{n+1}$ defined as follows is non-singular, where $T_{1} \mathbb{R}^{n+1}$ means the unit tangent bundle of $\mathbb{R}^{n+1}$.

$$
L_{f}(x)=\left(f(x), \nu_{f}(x)\right) .
$$

It is wll-known that the germ of spherical wave front $\widetilde{\Phi}_{t}:\left(S^{n}, \theta\right) \rightarrow$ $S_{N,+}^{n+1}-\{N\}$ is Legendrian for any real number $t(-\pi<t<\pi)$ and any $\theta \in S^{n}$. Some Legendrian map-germs are closely related with pedal hypersurface germs $([57,58])$. For more details on Legendrian mapgerms, for example see $[2,4,5,31,32,33]$. 


\section{$\S 3$. Several results obtained by using the spherical method}

\subsection{Crystallines}

Let $\gamma: S^{n} \rightarrow \mathbb{R}_{+}$be a continuous function. Recall that, in Subsection 2.5, the spherical Wulff shape $\widetilde{\mathcal{W}}_{\gamma}$ associated with the Wulff shape $\mathcal{W}_{\gamma}$ was defined as follows.

$$
\widetilde{\mathcal{W}}_{\gamma}=\alpha_{N}^{-1} \circ \operatorname{Id}\left(\mathcal{W}_{\gamma}\right)
$$

Moreover, the spherical dual $\mathcal{D} \widetilde{\mathcal{W}}_{\gamma}$ of $\widetilde{\mathcal{W}}_{\gamma}$ was defined as follows.

$$
\mathcal{D} \widetilde{\mathcal{W}}_{\gamma}=\left(\widetilde{\mathcal{W}}_{\gamma}\right)^{\circ}=\left(\alpha_{N}^{-1} \circ \operatorname{Id}\left(\mathcal{W}_{\gamma}\right)\right)^{\circ}
$$

As explained in Subsection 2.5, the dual Wulff shape $\mathcal{D} \mathcal{W}_{\gamma}$ is expressed in terms of $\mathcal{D} \widetilde{\mathcal{W}}_{\gamma}$ as follows.

$$
\mathcal{D} \mathcal{W}_{\gamma}=I d^{-1} \circ \alpha_{N}\left(\mathcal{D} \widetilde{\mathcal{W}}_{\gamma}\right)=I d^{-1} \circ \alpha_{N}\left(\left(\alpha_{N}^{-1} \circ \operatorname{Id}\left(\mathcal{W}_{\gamma}\right)\right)^{\circ}\right) .
$$

A convex integrand $\gamma$ is called a crystalline if the Wulff shape $\mathcal{W}_{\gamma}$ is a polytope. Notice that, inside $S^{n+1}$, we have Maehara's lemma (Lemma 2.6) for the spherical polar operation; and Maehara's lemma works very well for spherical polytopes. Thus, thanks to Maehara's lemma, we relatively easily have the following theorem.

Theorem 1 ([60]). Let $\gamma: S^{n} \rightarrow \mathbb{R}_{+}$be a continuous function. Then, $\gamma$ is a crystalline if and only if its dual convex integrand $\delta$ is a crystalline.

Since it is easy to see that the dual Wulff shape of $\mathcal{W}_{\gamma}$ is exactly the convex hull of $\frac{1}{\gamma}$ polar plot, it follows that the dual Wulff shape of $\mathcal{W}_{\gamma}$ may be regarded as a generalization of Frank-Meijering construction (for the Frank-Meijering construction, see [18, 48, 61]).

Although the proof of Theorem 1 is relatively easy, Theorem 1 is useful. For instance, the following result may be regarded as one of applications of Theorem 1.

Theorem 2 ([60]). Let $\gamma: S^{n} \rightarrow \mathbb{R}_{+}$be a function of class $C^{1}$. Then the Wulff shape $\mathcal{W}_{\gamma}$ is never a polytope.

For the proof of Theorem 2, we use not only the spherical method and Theorem 1 but also the fact that the boundary of the convex hull of the graph of a $C^{1}$ function $S^{n} \rightarrow \mathbb{R}_{+}$is a $C^{1}$ submanifold (for this fact, see $[65,74])$. 


\subsection{Aperture of plane curves}

Let $\mathbf{r}: S^{1} \rightarrow \mathbb{R}^{2}$ be a $C^{\infty}$ immersion. For the $\mathbf{r}$, we consider its inside region $\mathcal{N} \mathcal{S}_{\mathbf{r}}$.

$$
\mathcal{N} \mathcal{S}_{\mathbf{r}}=\mathbb{R}^{2}-\bigcup_{s \in S^{1}}\left(\mathbf{r}(s)+d \mathbf{r}_{s}\left(T_{s}\left(S^{1}\right)\right)\right)
$$

Since the perspective projection of $\mathbf{r}\left(S^{1}\right)$ from any point of the inside region is non-singular, $\mathcal{N} \mathcal{S}_{\mathbf{r}}$ is called no-silhouette of $\mathbf{r}$.

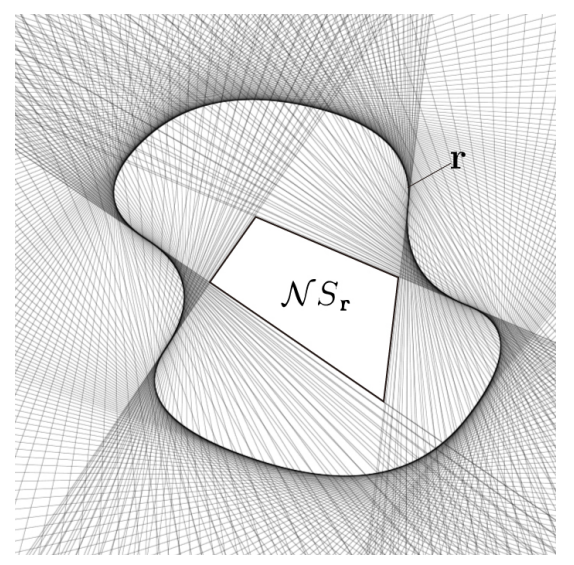

Fig. 14. Taken from [35]. The no-silhouette $\mathcal{N S}_{\mathbf{r}}$.

Next rotate all tangent lines about their tangent points simultaneously with the same angle, and consider the following inside region.

$$
\mathcal{N} \mathcal{S}_{\theta, \mathbf{r}}=\mathbb{R}^{2}-\bigcup_{s \in S^{1}}\left(\mathbf{r}(s)+R_{\theta}\left(d \mathbf{r}_{s}\left(T_{s}\left(S^{1}\right)\right)\right)\right)
$$

where $R_{\theta}: \mathbb{R}^{2} \rightarrow \mathbb{R}^{2}$ is the rotation defined by $R_{\theta}(x, y)=(x \cos \theta-$ $y \sin \theta, x \sin \theta+y \cos \theta)$.

The aperture of $S^{1}$ is the animation

$$
\left.\left(\bigcup_{0 \leq \theta<\pi / 2}\left(\mathcal{N} \mathcal{S}_{\theta, \mathbf{r}} \times\{\theta\}\right)\right) \bigcup(\{(0,0)\} \times\{\pi / 2)\}\right) \subset \mathbb{R}^{2} \times \mathbb{R}
$$

We are interested in how the topological closure of $\mathcal{N} \mathcal{S}_{\theta, \mathbf{r}}$ is growing as $\theta$ increases for general $\mathbf{r}: S^{1} \rightarrow \mathbb{R}^{2}$ such that $\mathcal{N S}_{\mathbf{r}} \neq \emptyset$. 


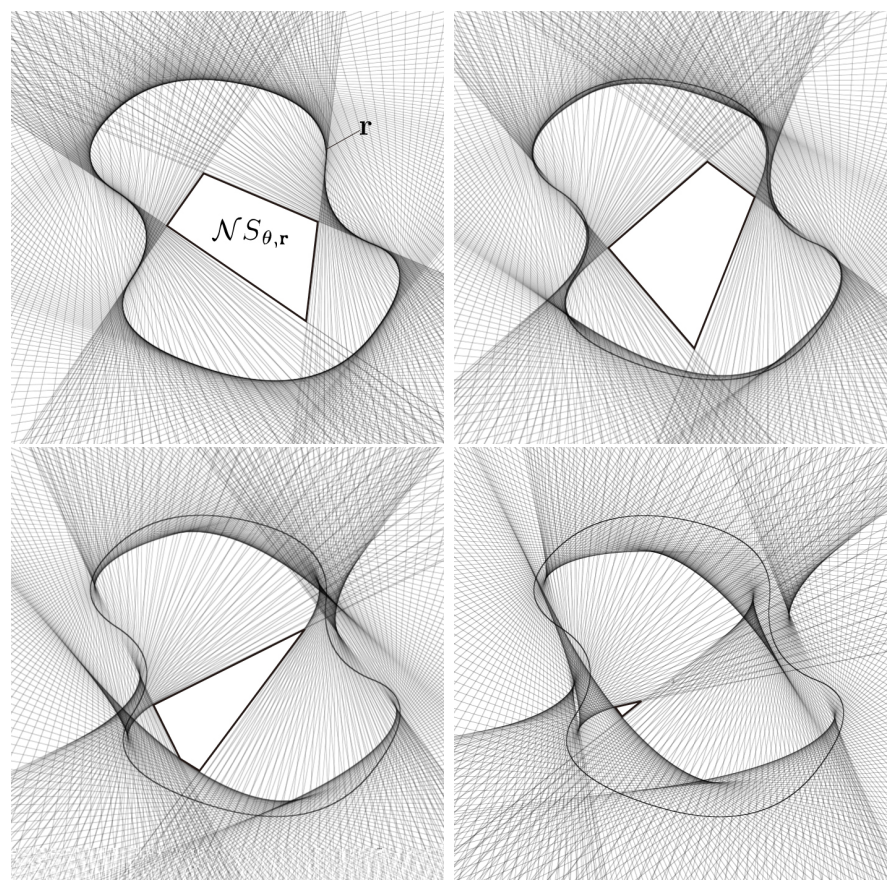

Fig. 15. Taken from [35]. Left top : $\theta=0$, right top : $\theta=$ $\pi / 12$, left bottom : $\theta=\pi / 6$, right bottom : $\theta=\pi / 4$.

Lemma 3.1 ([35]). For any $C^{\infty}$ immersion $\mathbf{r}: S^{1} \rightarrow \mathbb{R}^{2}, \mathcal{N S} \mathcal{S}_{\frac{\pi}{2}, \mathbf{r}}$ is the empty set.

Since our object is moving convex bodies (and its limit) $\overline{\mathcal{N} \mathcal{S}_{\theta, \mathbf{r}}}$, it is better to treat the spherical polar operation as a transform of a space consisting of reasonable subsets of $S^{2}$. Let $\mathcal{H}\left(S^{n+1}\right)$ be the set consisting of non-empty closed subsets of $S^{n+1}$. It is well-known that $\mathcal{H}\left(S^{n+1}\right)$ is a complete metric space with respect to the Pompeiu-Hausdorff metric (see $[6,15])$. Let $\mathcal{H}^{\circ}\left(S^{n+1}\right)$ be the subspace of $\mathcal{H}\left(S^{n+1}\right)$ consisting of non-empty closed subset $\widetilde{W}$ of $S^{n+1}$ such that $\widetilde{W}^{\circ} \neq \emptyset$.

$$
\mathcal{H}^{\circ}\left(S^{n+1}\right)=\left\{\widetilde{W} \in \mathcal{H}\left(S^{n+1}\right) \mid \widetilde{W}^{\circ} \neq \emptyset\right\} .
$$

Then, the spherical polar transform

$$
\bigcirc: \mathcal{H}^{\circ}\left(S^{n+1}\right) \rightarrow \mathcal{H}^{\circ}\left(S^{n+1}\right)
$$


is defined as follows.

$$
\bigcirc(\widetilde{W})=\widetilde{W}^{\circ}
$$

By Lemma 2.3, it follows that $\widetilde{W}^{\circ}$ is contained in $\mathcal{H}^{\circ}\left(S^{n+1}\right)$ for any $\widetilde{W} \in \mathcal{H}^{\circ}\left(S^{n+1}\right)$. Thus, the spherical polar transform $\bigcirc$ is well-defined. The following proposition is proved in [35].

Proposition $10([35])$. In the case $n=1$, the spherical polar transform $\bigcirc: \mathcal{H}^{\circ}\left(S^{2}\right) \rightarrow \mathcal{H}^{\circ}\left(S^{2}\right)$ is continuous.

It is easily seen that Proposition 10 holds for any $n \in \mathbb{N}$. In the next subsection, a result obtained in [22] which is stronger than Proposition 10 (for general $n$ ) is surveyed. Namely, in Subsection 3.3, it shall be stated that the spherical polar transform $\bigcirc: \mathcal{H}^{\circ}\left(S^{n+1}\right) \rightarrow \mathcal{H}^{\circ}\left(S^{n+1}\right)$ is Lipschitz.

By using Proposition 10, the following is obtained.

Proposition 11 ([35]). With respect to the Pompeiu-Hausdorff metric, the topological closure of $\mathcal{N S}_{\theta, \mathbf{r}}$ varies continuously depending on $\theta$ while $\mathcal{N} \mathcal{S}_{\theta, \mathbf{r}}$ is not empty.

Therefore, by Lemma 3.1, the notion of aperture angle $\theta_{\mathbf{r}}\left(0<\theta_{\mathbf{r}} \leq\right.$ $\pi / 2)$ is well-defined as follows.

Definition 12 ([35]). Let $\mathbf{r}: S^{1} \rightarrow \mathbb{R}^{2}$ be a $C^{\infty}$ immersion with its no-silhouette $\mathcal{N S}_{\mathbf{r}} \neq \emptyset$. Then, $\theta_{\mathbf{r}}\left(0<\theta_{\mathbf{r}} \leq \frac{\pi}{2}\right)$ is defined as the largest angle which satisfies $\mathcal{N} \mathcal{S}_{\theta, \mathbf{r}} \neq \emptyset$ for any $\theta\left(0 \leq \theta<\theta_{\mathbf{r}}\right)$. The angle $\theta_{\mathbf{r}}$ is called the aperture angle of the given $\mathbf{r}$.

It is not difficult to see that $\overline{\mathcal{N} \mathcal{S}_{\theta, \mathbf{r}}}$ is a Wulff shape for any $\theta(0 \leq$ $\left.\theta<\theta_{\mathbf{r}}\right)$. Thus, we may regard that $\left\{\overline{\mathcal{N} \mathcal{S}_{\theta, \mathbf{r}}} \mid 0 \leq \theta \leq \theta_{\mathbf{r}}\right\}$ is a simple geometric model of crystal growth.

We are interested in how our simple geometric model of crystal growth melts as $\theta$ goes to $\theta_{\mathbf{r}}$ from 0 .

Theorem 3 ([35]). Let $\mathbf{r}: S^{1} \rightarrow \mathbb{R}^{2}$ be a $C^{\infty}$ immersion with its no-silhouette $\mathcal{N S}_{\mathbf{r}} \neq \emptyset$. Then, for any $\theta\left(0<\theta<\theta_{\mathbf{r}}\right), \overline{\mathcal{N} \mathcal{S}_{\theta}, \mathbf{r}}$ is never a polygon even if the given $\overline{\mathcal{N} \mathcal{S}_{\mathbf{r}}}$ is a polygon.

By Theorem 3, among $\overline{\mathcal{N} \mathcal{S}_{\frac{\pi}{12}}, \mathbf{r}}, \overline{\mathcal{N} \mathcal{S}_{\frac{\pi}{6}, \mathbf{r}}}$ and $\overline{\mathcal{N} \mathcal{S}_{\frac{\pi}{4}, \mathbf{r}}}$ in Figure 15 , there are no polygons; although $\overline{\mathcal{N} \mathcal{S}_{0, \mathrm{r}}}$ is a polygon constructed by four tangent lines to $\mathbf{r}$ at four inflection points.

Theorem 4 ([35]). Let $\mathbf{r}: S^{1} \rightarrow \mathbb{R}^{2}$ be a $C^{\infty}$ immersion with its nosilhouette $\mathcal{N S}_{\mathbf{r}} \neq \emptyset$. Then, there exists the unique point $P_{\mathbf{r}} \in \mathbb{R}^{2}$ such 
that for any sequence $\left\{\theta_{i}\right\}_{i=1,2, \ldots} \subset\left[0, \theta_{\mathbf{r}}\right)$ converging to the aperture angle $\theta_{\mathbf{r}}$, the following holds:

$$
\lim _{i \rightarrow \infty} d_{H}\left(\overline{\mathcal{N} \mathcal{S}_{\theta_{i}}, \mathbf{r}}, P_{\mathbf{r}}\right)=0 .
$$

Here, $d_{H}: \mathcal{H}\left(\mathbb{R}^{2}\right) \times \mathcal{H}\left(\mathbb{R}^{2}\right) \rightarrow \mathbb{R}$ is the Pompeiu-Hausdorff metric. Theorem 4 justifies the following definition.

Definition 13 ([35]). Let $\mathbf{r}: S^{1} \rightarrow \mathbb{R}^{2}$ be a $C^{\infty}$ immersion with its no-silhouette $\mathcal{N S}_{\mathbf{r}} \neq \emptyset$. Then, the unique point $P_{\mathbf{r}}=\lim _{\theta \rightarrow \theta_{\mathbf{r}}} \overline{\mathcal{N} \mathcal{S}_{\theta, \mathbf{r}}}$ is called the aperture point of $\mathbf{r}$.

The simplest example is a circle. The aperture point of it is its center. In this case, the aperture angle is $\pi / 2$. In general, in the case of curves with no inflection points, the crystal growth is relatively simpler than curves with inflections as follows.

Theorem 5 ([35]). Let $\mathbf{r}: S^{1} \rightarrow \mathbb{R}^{2}$ be a $C^{\infty}$ immersion with its no-silhouette $\mathcal{N S}_{\mathbf{r}} \neq \emptyset$. Suppose that $\mathbf{r}$ has no inflection points. Then, for any two $\theta_{1}, \theta_{2}$ satisfying $0 \leq \theta_{1}<\theta_{2}<\theta_{\mathbf{r}}$, the following inclusion holds:

$$
\mathcal{N} \mathcal{S}_{\theta_{1}, \mathbf{r}} \supset \mathcal{N} \mathcal{S}_{\theta_{2}, \mathbf{r}}
$$

Figure 15 shows that in general it is impossible to expect the same property as Theorem 5 for a curve with inflection points.

\subsection{The spherical polar transform}

In Subsection 3.1, the spherical polar set played an essential role for investigating a Wulff shape. In Subsection 3.2, moving convex bodies (and their limits) in $\mathbb{R}^{2}$ were investigated. One of essential tools in Subsection 3.2 was treating any spherical polar set as the image of a non-empty closed subset by the map called the spherical polar transform. In [22], the spherical polar transform is investigated more and more by using the spherical method explained in Section 2. In this subsection, we survey results on the spherical polar transform obtained in [22].

Recall that $\mathcal{H}^{\circ}\left(S^{n+1}\right)$ is the set consisting of non-empty closed subsets $\widetilde{W}$ of $S^{n+1}$ such that $\widetilde{W}^{\circ} \neq \emptyset$ and that the spherical polar transform $\bigcirc: \mathcal{H}^{\circ}\left(S^{n+1}\right) \rightarrow \mathcal{H}^{\circ}\left(S^{n+1}\right)$ is defined by $\bigcirc(\widetilde{W})=\widetilde{W}^{\circ}$.

Lemma 3.2. For any $n \in \mathbb{N}$, the mapping $\bigcirc: \mathcal{H}^{\circ}\left(S^{n+1}\right) \rightarrow$ $\mathcal{H}^{\circ}\left(S^{n+1}\right)$ is continuous.

As we already announced in Subsection 3.2, Lemma 3.2 can be improved as follows. 
Definition 14. Let $\left(X, d_{X}\right),\left(Y, d_{Y}\right)$ be metric spaces. A mapping $f: X \rightarrow Y$ is said to be Lipschitz if there exists a positive real number $K \in \mathbb{R}_{+}$such that the following holds for any $x_{1}, x_{2} \in X$ :

$$
d_{Y}\left(f\left(x_{1}\right), f\left(x_{2}\right)\right) \leq K d_{X}\left(x_{1}, x_{2}\right) .
$$

The positive real number $K \in \mathbb{R}_{+}$for a Lipschitz mapping is called the Lipschitz coefficient of $f$.

Proposition $12([22])$. Let $n$ be a positive integer. Then, the spherical polar transform $\bigcirc: \mathcal{H}^{\circ}\left(S^{n+1}\right) \rightarrow \mathcal{H}^{\circ}\left(S^{n+1}\right)$ is Lipschitz with respect to the Pompeiu-Hausdorff metric.

Proposition 12 suggests that the spherical polar transform $\bigcirc$ : $\mathcal{H}^{\circ}\left(S^{n+1}\right) \rightarrow \mathcal{H}^{\circ}\left(S^{n+1}\right)$ might have many nice properties. However, it is easily seen that $\bigcirc$ is neither injective nor surjective. Hence, in order to obtain nice properties of the spherical polar transform, we naturally reach restrictions of $\bigcirc$ to reasonable subspaces of $\mathcal{H}^{\circ}\left(S^{n+1}\right)$.

Definition 15. (1) A subset $\widetilde{W}$ of $S^{n+1}$ is called a spherical convex body if $\widetilde{W}$ is hemispherical, closed and spherical convex and it has an interior point.

(2) For any point $P$ of $S^{n+1}$, let $\mathcal{H}_{\text {Wulff }}\left(S^{n+1}, P\right)$ be the following set:

$$
\begin{gathered}
\mathcal{H}_{\text {Wulff }}\left(S^{n+1}, P\right)=\left\{\widetilde{W} \in \mathcal{H}\left(S^{n+1}\right) \mid \widetilde{W} \cap H(-P)=\emptyset, P \in \operatorname{int}(\widetilde{W}),\right. \\
\widetilde{W} \text { is a spherical convex body }\}
\end{gathered}
$$

where $\operatorname{int}(\widetilde{W})$ stands for the set consisting of interior points of $\widetilde{W}$. The topological closure of $\mathcal{H}_{\text {Wulff }}\left(S^{n+1}, P\right)$ is denoted by $\overline{\mathcal{H}_{\text {Wulff }}\left(S^{n+1}, P\right)}$.

(3) Let $\mathcal{H}_{\mathrm{S}-\mathrm{conv}}\left(S^{n+1}\right)$ be the set consisting of spherical convex closed sets. The topological closure of $\mathcal{H}_{\mathrm{S}-\mathrm{conv}}\left(S^{n+1}\right)$ is denoted by $\overline{\mathcal{H}_{\mathrm{S}-\mathrm{conv}}\left(S^{n+1}\right)}$.

Recall that $N \in S^{n+1}$ stands for the north pole $=(0, \ldots, 0,1) \in S^{n+1}$. Notice that in the case $P=N, \mathcal{H}_{\text {Wulff }}\left(S^{n+1}, N\right)$ is nothing but the set consisting of spherical Wulff shapes defined in Subsection 2.5. Thus, we have the following characterization.

$$
\mathcal{H}_{\text {Wulff }}\left(S^{n+1}, N\right)=\left\{\widetilde{\mathcal{W}}_{\gamma}=\alpha_{N}^{-1} \circ \operatorname{Id}\left(\mathcal{W}_{\gamma}\right) \mid \gamma \in C^{0}\left(S^{n}, \mathbb{R}_{+}\right)\right\}
$$

The next proposition explains the relation between $\overline{\mathcal{H}_{\text {Wulff }}\left(S^{n+1}, P\right)}$ and $\overline{\mathcal{H}_{\mathrm{S}-\mathrm{conv}}\left(S^{n+1}\right)}$. 
Proposition 13 ([22]).

$$
\bigcup_{P \in S^{n+1}} \overline{\mathcal{H}_{\text {Wulff }}\left(S^{n+1}, P\right)}=\overline{\mathcal{H}_{\mathrm{S}-\operatorname{conv}}\left(S^{n+1}\right)} .
$$

Proposition $14([22]) . \quad$ (1) Let $P$ be a point of $S^{n+1}$. Then, the set $\overline{\mathcal{H}_{\text {Wulff }}\left(S^{n+1}, P\right)}$ is contained in $\mathcal{H}^{\circ}\left(S^{n+1}\right)$.

(2) For any point $P \in S^{n+1}$, the following holds:

$$
\bigcirc\left(\mathcal{H}_{\text {Wulff }}\left(S^{n+1}, P\right)\right)=\mathcal{H}_{\text {Wulff }}\left(S^{n+1}, P\right) .
$$

(3) For any point $P \in S^{n+1}$, the following holds:

$$
\bigcirc\left(\overline{\mathcal{H}_{\text {Wulff }}\left(S^{n+1}, P\right)}\right)=\overline{\mathcal{H}_{\text {Wulff }}\left(S^{n+1}, P\right)} .
$$

(4) For any point $P \in S^{n+1}$, the following restriction of $\bigcirc$ is injective:

$\left.\bigcirc\right|_{\overline{\mathcal{H}_{\text {Wulff }}\left(S^{n+1}, P\right)}}: \overline{\mathcal{H}_{\text {Wulff }}\left(S^{n+1}, P\right)} \rightarrow \overline{\mathcal{H}_{\text {Wulff }}\left(S^{n+1}, P\right)}$.

(5) $\overline{\mathcal{H}_{\mathrm{S}-\mathrm{conv}}\left(S^{n+1}\right)} \subset \mathcal{H}^{\circ}\left(S^{n+1}\right)$.

(6) $\bigcirc\left(\mathcal{H}_{\text {S-conv }}\left(S^{n+1}\right)\right) \neq \mathcal{H}_{\text {S-conv }}\left(S^{n+1}\right)$.

(7) $\bigcirc\left(\overline{\mathcal{H}_{\text {S-conv }}\left(S^{n+1}\right)}\right)=\overline{\mathcal{H}_{\text {S-conv }}\left(S^{n+1}\right)}$.

(8) The restriction of $\bigcirc$ to $\overline{\mathcal{H}_{\mathrm{S}-\mathrm{conv}}\left(S^{n+1}\right)}$ is injective.

By Proposition 14, we have the following:

Lemma 3.3 ([22]). Each of the following is well-defined bijective mapping.

$$
\begin{aligned}
& \left.\right|_{\mathcal{H}_{\text {Wulff }}\left(S^{n+1}, P\right)}: \mathcal{H}_{\text {Wulff }}\left(S^{n+1}, P\right) \rightarrow \mathcal{H}_{\text {Wulff }}\left(S^{n+1}, P\right), \\
& \left.\bigcirc\right|_{\overline{\mathcal{H}_{\text {Wulff }}\left(S^{n+1}, P\right)}:}: \overline{\mathcal{H}_{\text {Wulff }}\left(S^{n+1}, P\right)} \rightarrow \overline{\mathcal{H}_{\text {Wulff }}\left(S^{n+1}, P\right)}, \\
& \bigcirc \overline{\mathcal{H}_{\mathrm{S}-\mathrm{conv}}\left(S^{n+1}\right)}: \overline{\mathcal{H}_{\mathrm{S}-\mathrm{conv}}\left(S^{n+1}\right)} \rightarrow \overline{\mathcal{H}_{\mathrm{S}-\operatorname{conv}}\left(S^{n+1}\right)} \text {. }
\end{aligned}
$$

Definition 16. Let $\left(X, d_{X}\right),\left(Y, d_{Y}\right)$ be metric spaces.

(1) A mapping $f: X \rightarrow Y$ is said to be bi-Lipschitz if $f$ is bijective and there exist positive real numbers $K, L \in \mathbb{R}_{+}$such that the following hold for any $x_{1}, x_{2} \in X$ and any $y_{1}, y_{2} \in Y$ :

$$
\begin{aligned}
d_{Y}\left(f\left(x_{1}\right), f\left(x_{2}\right)\right) & \leq K d_{X}\left(x_{1}, x_{2}\right), \\
d_{X}\left(f^{-1}\left(y_{1}\right), f^{-1}\left(y_{2}\right)\right) & \leq L d_{Y}\left(y_{1}, y_{2}\right),
\end{aligned}
$$


(2) A mapping $f: X \rightarrow Y$ is called an isometry if $f$ is bijective and the following holds for any $x_{1}, x_{2} \in X$ :

$$
d_{Y}\left(f\left(x_{1}\right), f\left(x_{2}\right)\right)=d_{X}\left(x_{1}, x_{2}\right) .
$$

Theorem 6 ([22]). Let $P$ be a point of $S^{n+1}$. Then, with respect to the Pompeiu-Hausdorff metric, the following two hold:

(1) The restriction of $\bigcirc$ to $\mathcal{H}_{\text {Wulff }}\left(S^{n+1}, P\right)$

$$
\left.\bigcirc\right|_{\mathcal{H}_{\text {Wulff }}\left(S^{n+1}, P\right)}: \mathcal{H}_{\text {Wulff }}\left(S^{n+1}, P\right) \rightarrow \mathcal{H}_{\text {Wulff }}\left(S^{n+1}, P\right)
$$

is an isometry.

(2) The restriction of $\bigcirc$ to $\overline{\mathcal{H}_{\text {Wulff }}\left(S^{n+1}, P\right)}$

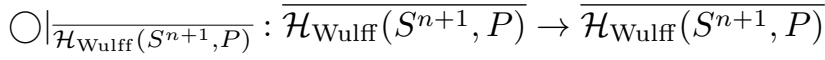

is an isometry.

For any positive real number $r$, let $D_{r}$ be the set consisting of $x \in \mathbb{R}^{n+1}$ satisfying $\|x\| \leq r$. Then, $D_{r}$ is a Wulff shape for any $r \in \mathbb{R}(r>0)$ and it is well-known that the dual Wulff shape of $D_{r}$ is $D_{\frac{1}{r}}$. Moreover, it is easily seen that $h\left(D_{r_{1}}, D_{r_{2}}\right)=\left|r_{1}-r_{2}\right|$ holds for any $r_{1}, r_{2} \in \mathbb{R}$ $\left(r_{1}, r_{2}>0\right)$, where $h$ is the Pompeiu-Hausdorff metric. Thus, it is impossible to expect the Euclidean counterpart of the assertion (1) of Theorem 6 . This shows an advantage of studying the spherical version of Wulff shapes. Moreover, the Euclidean counterpart of $\overline{\mathcal{H}_{\text {Wulff }}\left(S^{n+1}, P\right)}$ is not well-defined. This, too, shows an advantage of studying the spherical version of Wulff shapes.

Theorem 7 ([22]). With respect to the Pompeiu-Hausdorff metric, the restriction of the spherical polar transform

$$
\left.\bigcirc\right|_{\overline{\mathcal{H}_{\mathrm{S}-\operatorname{conv}}\left(S^{n+1}\right)}}: \overline{\mathcal{H}_{\mathrm{S}-\operatorname{conv}}\left(S^{n+1}\right)} \rightarrow \overline{\mathcal{H}_{\mathrm{S}-\operatorname{conv}}\left(S^{n+1}\right)}
$$

is bi-Lipschitz but never an isometry.

\subsection{Convex integrands of class $C^{1}$}

Recall that the following mapping

$$
\mathcal{W}: C^{0}\left(S^{n}, \mathbb{R}_{+}\right) \rightarrow \mathcal{H}_{\text {conv }, \mathbf{0}}\left(\mathbb{R}^{n+1}\right)
$$

defined by $\mathcal{W}(\gamma)=\mathcal{W}_{\gamma}$ has been introduced and properties of $\mathcal{W}$ has been studied in Section 1. Recall that $\mathcal{W}$ is surjective. Thus, for any 
convex body $W \subset \mathbb{R}^{n+1}$ containing the origin of $\mathbb{R}^{n+1}$ as an interior point of $W$, it follows that

$$
\mathcal{W}^{-1}(W) \neq \emptyset .
$$

Recall moreover that there uniquely exists the most efficient $C^{0}$ function, called the convex integrand of $W$ and denoted by $\gamma_{W}$. By using the spherical method explained in Section 2, we can obtain a characterization of a $C^{1}$ convex integrand as follows.

Theorem 8 ([23]). Let $W \subset \mathbb{R}^{n+1}$ be a convex body containing the origin of $\mathbb{R}^{n+1}$ as an interior point of $W$. Then, $W$ is strictly convex if and only if its convex integrand $\gamma_{W}$ is of class $C^{1}$.

Remarks should be stated on Theorem 8. The first remark concerns [52]. Thanks to [52], the authors learned that Frank Morgan has shown a result in [51] which is similar as Theorem 8. His result is the following Theorem 9.

Definition 17. (1) Let $W$ be a strictly convex convex body in $\mathbb{R}^{n+1}$ containing the origin of $\mathbb{R}^{n+1}$ as its interior point. Then, $W$ is said to be uniformly convex if the mapping $f: S^{n} \rightarrow S^{n}$ defined by $f(\mathbf{n})=\theta$ is Lipschitz, where $\mathbf{n} \in S^{n}$ is the unit normal vector of $\partial W$ at the intersection $\mathbb{R}_{+} \theta \cap \partial W$.

(2) A continuous function $\gamma: S^{n} \rightarrow \mathbb{R}_{+}$is said to be of class $C^{1,1}$ if it is of class $C^{1}$ and all first partial derivatives are Lipschitz.

Theorem 9 ([51]). Let $W \subset \mathbb{R}^{n+1}$ be a convex body containing the origin of $\mathbb{R}^{n+1}$ as an interior point of $W$. Then, $W$ is uniformly convex if and only if its convex integrand $\gamma_{W}$ is of class $C^{1,1}$.

As Figure 16 shows, the notion of "uniform convexity"(resp., "class $C^{1,1 ")}$ is actually stronger than "strict convexity" (resp., "class $C^{1 "}$ ). Moreover, "strict convexity" (resp., "class $C^{1 "}$ ") is much more common, natural and easy to treat than "uniform convexity" (resp., "class $C^{1,1}$ "). Thus, Theorem 8 is a more applicable and more desirable result than Theorem 9 .

The next remark is that Theorem 8 is much stronger than Theorem 2 given in Subsection 3.1. This is explained as follows. Suppose that a $C^{1}$ function $\gamma: S^{1} \rightarrow \mathbb{R}_{+}$is given. Then, since inv: $\mathbb{R}^{n+1}-\{\mathbf{0}\} \rightarrow \mathbb{R}^{n+1}-\{\mathbf{0}\}$ is a $C^{\infty}$ diffeomorphism, it follows that the boundary of the convex hull of $\operatorname{inv}(\operatorname{graph}(\gamma))$ is the graph of a $C^{1}$ function $S^{n} \rightarrow \mathbb{R}_{+}$(for instance, refer to $[65,74])$. By definition, we have that the convex integrand $\gamma_{w_{\gamma}}$ is of class $C^{1}$. Therefore, by Theorem 8 , the Wulff shape $\mathcal{W}_{\gamma}$ must be 

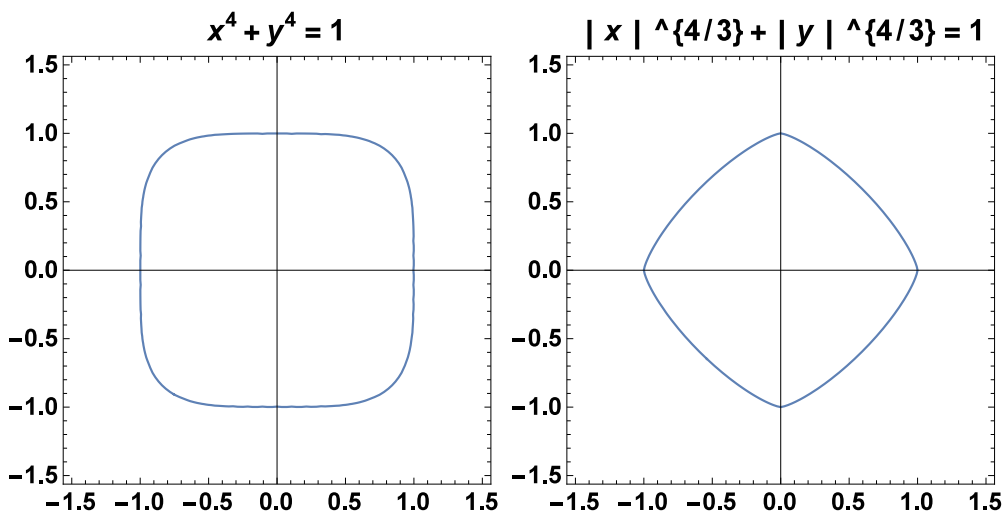

Fig. 16. These two examples are taken from [51]. The convex hull of left (resp., right) curve is called the unit $L_{4}$ (resp., $L_{4 / 3}$ ) ball. The unit $L_{4}$ ball is not uniformly convex but strictly convex and has $C^{1,1}$ boundary. On the other hand, the unit $L_{4 / 3}$ ball does not have $C^{1,1}$ boundary but does have $C^{1}$ boundary and is uniformly convex.

strictly convex. Hence, $\mathcal{W}_{\gamma}$ is never a polytope. Theorem 8 asserts that even the converse holds if $\gamma$ is a convex integrand. Thus, Theorem 8 is much stronger than Theorem 2 .

Moreover, Theorem 8 has many applications. For instance, we have the following as a direct application.

Corollary 1 ([23]). A Wulff shape in $\mathbb{R}^{n+1}$ is strictly convex if and only if the boundary of its dual Wulff shape is $C^{1}$ diffeomorphic to $S^{n}$.

In particular, we have the following:

Corollary 2 ([23]). A Wulff shape in $\mathbb{R}^{n+1}$ is strictly convex and its boundary is $C^{1}$ diffeomorphic to $S^{n}$ if and only if its dual Wulff shape is strictly convex and the boundary of it is $C^{1}$ diffeomorphic to $S^{n}$.

It seems interesting to compare Corollary 2 and Theorem 1.

There is also an application of Theorem 8 from the viewpoint of pedal. Firstly, we recall the definition of pedal given in Subsection 2.1.

Definition 2. Let $\Phi: S^{n} \rightarrow \mathbb{R}^{n+1}-\{\mathbf{0}\}$ be a $C^{1}$ embedding. Then, the pedal relative to the pedal point $\mathbf{0}$ for $\Phi$, denoted by $\operatorname{ped}_{\Phi, \mathbf{0}}: S^{n} \rightarrow$ $\mathbb{R}^{n+1}$, is the mapping which maps $\theta \in S^{n}$ to the unique nearest point of 
$\Phi(\theta)+T_{\Phi(\theta)} \Phi\left(S^{n}\right)$ from the origin $\mathbf{0}$, where $\Phi(\theta)+T_{\Phi(\theta)} \Phi\left(S^{n}\right)$ stands for the affine tangent hyperplane to $\Phi\left(S^{n}\right)$ at $\Phi(\theta)$

Let $W \subset \mathbb{R}^{n+1}$ be a Wulff shape. Suppose that $\partial W$ is $C^{1}$ diffeomorphic to $S^{n}$. Then, $\partial W$ may be regarded as the image of a certain $C^{1}$ embed$\operatorname{ding} F: S^{n} \rightarrow \mathbb{R}^{n+1}-\{\mathbf{0}\}$, and $\gamma_{W}$ is exactly the pedal of $\partial W$ relative to the origin. Theorem 8 gives a sufficient condition for the pedal of $\partial W$ relative to the origin to be smooth:

Corollary 3 ([23]). Suppose that a Wulff shape $W$ in $\mathbb{R}^{n+1}$ is strictly convex and its boundary is $C^{1}$ diffeomorphic to $S^{n}$. Then, $\operatorname{ped}_{\Phi, \mathbf{0}}: S^{n} \rightarrow \mathbb{R}^{n+1}$ is of class $C^{1}$.

\subsection{Wulff shapes with $C^{1}$ boundary}

The following result is an application of Theorem 8 as well. Thus, Theorem 10, too, may be regarded as a result of the spherical method.

Theorem 10. Let $\gamma: S^{n} \rightarrow \mathbb{R}_{+}$be a continuous function and let $\mathcal{W}_{\gamma}$ be the Wulff shape associated with $\gamma$. Suppose that the boundary of $\mathcal{W}_{\gamma}$ is a $C^{1}$ submanifold. Then, $\gamma$ must be the convex integrand of $\mathcal{W}_{\gamma}$. In other words, the following holds.

$$
\mathcal{W}^{-1}\left(\mathcal{W}_{\gamma}\right)=\{\gamma\}
$$

where $\mathcal{W}: C^{0}\left(S^{n}, \mathbb{R}_{+}\right) \rightarrow \mathcal{H}_{\text {conv }, \mathbf{0}}\left(\mathbb{R}^{n+1}\right)$. is the mapping defined by $\mathcal{W}(\gamma)=\mathcal{W}_{\gamma}$

Theorem 10 asserts that the given continuous function $\gamma$ is uniquely determined only by the shape of crystal if the boundary of $\mathcal{W}_{\gamma}$ is of class $C^{1}$. In other words, Theorem 10 is equivalet to say that the following inclusion holds, where $C I\left(S^{n}, \mathbb{R}_{+}\right)$is the subset of $C^{0}\left(S^{n}, \mathbb{R}_{+}\right)$consisting of convex integrands and $\mathcal{H}_{\text {conv, } \mathbf{0}}^{1}\left(\mathbb{R}^{n+1}\right)$ is the set of convex bodies $W$ in $\mathbb{R}^{n+1}$ such that the origin of $\mathbb{R}^{n+1}$ is an interior point of $W$ and the boundary of $W$ is a $C^{1}$ submanifold.

$$
C I\left(S^{n}, \mathbb{R}_{+}\right) \supset \mathcal{W}^{-1}\left(\mathcal{H}_{\text {conv }, \mathbf{0}}^{1}\left(\mathbb{R}^{n+1}\right)\right) .
$$

The converse of Theorem 10 does not hold in general except for $n=1$.

Proof. Define $\gamma_{\mathcal{w}_{\gamma}}: S^{n} \rightarrow \mathbb{R}_{+}$so that the graph of $\gamma_{\mathcal{w}_{\gamma}}$ satisfies the following:

$$
\Gamma_{\gamma}=\operatorname{inv}\left(\operatorname{graph}\left(\gamma_{\mathcal{W}_{\gamma}}\right)\right)
$$

Here, as defined in Section 1, $\Gamma_{\gamma}$ is the boudary of the convex hull of $\operatorname{inv}(\operatorname{graph}(\gamma))$. Then, $\gamma_{\mathcal{W}_{\gamma}}$ is the convex integrand of $\mathcal{W}_{\gamma}$, and the Wulff shape associated with $\gamma_{\mathcal{W}_{\gamma}}$ is exactly $\mathcal{W}_{\gamma}$. 
Suppose that $\gamma_{\mathcal{w}_{\gamma}} \neq \gamma$. Then, there exists at least one point $x \in \Gamma_{\gamma}$ such that $x \notin \operatorname{inv}(\operatorname{graph}(\gamma))$. Since $\Gamma_{\gamma}$ is the boundary of the convex hull of $\operatorname{inv}(\operatorname{graph}(\gamma))$, this implies that there exist two distinct points $x_{1}, x_{2}$ in $\Gamma_{\gamma} \cap \operatorname{inv}(\operatorname{graph}(\gamma))$ and a real number $t(0<t<1)$ such that $x=(1-t) x_{1}+t x_{2}$.

On the other hand, since the boundary of $\mathcal{W}_{\gamma}$ is a $C^{1}$ submanifold, by Theorem 8 , the convex hull of $\operatorname{inv}(\operatorname{graph}(\gamma))$ must be strictly convex. Hence, we have a contradiction.

Q.E.D.

\subsection{Self-dual Wulff shapes and spherical Wulff shapes of constant width $\pi / 2$}

Recall that a subset $\widetilde{W}$ of $S^{n+1}$ is called a spherical convex body if $\widetilde{W}$ is closed and spherical convex and it has an interior point (see Definition 7 in Subsection 2.3).

Definition 18. Let $\widetilde{W} \subset S^{n+1}$ be a spherical convex body. Then, a hemisphere $H(P)$ is said to support $\widetilde{W}$ if $H(P)$ contains $\widetilde{W}$ and there exists a point $Q$ of the boundary of $\widetilde{W}$ which is contained in the boundary of $H(P)$.

For a spherical convex body $\widetilde{W}$ and a hemisphere $H(P)$ supporting $\widetilde{W}$, following [37, 38], the width of $\widetilde{W}$ determined by $H(P)$ is defined as follows. For any two $P, Q \in S^{n+1}(P \neq \pm Q)$, the intersection $H(P) \cap$ $H(Q)$ is called a lune of $S^{n+1}$. The thickness of the lune $H(P) \cap H(Q)$, denoted by $\triangle(H(P) \cap H(Q))$, is the real number $\pi-|P Q|$, where $|P Q|$ stands for the length of the arc $P Q$. For a spherical convex body $\widetilde{W}$ and a hemisphere $H(P)$ supporting $\widetilde{W}$, the width of $\widetilde{W}$ determined by $H(P)$, denoted by width ${ }_{H(P)} \widetilde{W}$, is the minimum of the following set:

$$
\{\triangle(H(P) \cap H(Q)) \mid \widetilde{W} \subset H(P) \cap H(Q), H(Q) \text { supports } \widetilde{W}\} .
$$

For any $\rho \in \mathbb{R}_{+}$less than $\pi$, a spherical convex body $\widetilde{W} \subset S^{n+1}$ is said to be of constant width $\rho$ if width $_{H(P)} \widetilde{W}=\rho$ for any $H(P)$ supporting $\widetilde{W}$.

Let $\mathcal{W}_{\gamma} \subset \mathbb{R}^{n+1}$ be a Wulff shape. In Subsection 2.5, the following set was called the spherical Wulff shape induced by $\mathcal{W}_{\gamma}$.

$$
\widetilde{\mathcal{W}}_{\gamma}=\alpha_{N}^{-1} \circ \operatorname{Id}\left(\mathcal{W}_{\gamma}\right) .
$$

Here, $I d: \mathbb{R}^{n+1} \rightarrow \mathbb{R}^{n+1} \times\{1\} \subset \mathbb{R}^{n+2}$ is the mapping defined by $\operatorname{Id}(x)=(x, 1), N \in S^{n+1}$ is the north pole of $S^{n+1}$ and $\alpha_{N}: S^{n+1}-$ 
$H(-N) \rightarrow \mathbb{R}^{n+1} \times\{1\} \subset \mathbb{R}^{n+2}$ is the central projection defined as follows.

$$
\begin{gathered}
\alpha_{N}\left(P_{1}, \ldots, P_{n+1}, P_{n+2}\right)=\left(\frac{P_{1}}{P_{n+2}}, \ldots, \frac{P_{n+1}}{P_{n+2}}, 1\right) \\
\left(\forall\left(P_{1}, \ldots, P_{n+1}, P_{n+2}\right) \in S^{n+1}-H(-N)\right) .
\end{gathered}
$$

Then, for any $\mathcal{W}_{\gamma}$, it is clear that the spherical Wulff shape induced by $\mathcal{W}_{\gamma}$ is a spherical convex body.

Definition 19. A Wulff shape $\mathcal{W}_{\gamma}$ is said to be self-dual if the following equality holds.

$$
\widetilde{\mathcal{W}}_{\gamma}=\mathcal{D} \widetilde{\mathcal{W}}_{\gamma}=\left(\widetilde{\mathcal{W}}_{\gamma}\right)^{\circ}
$$

Theorem 11 ([24]). Let $\gamma: S^{n} \rightarrow \mathbb{R}_{+}$be a continuous function. Then, the Wulff shape $W_{\gamma}$ is self-dual if and only if the spherical Wulff shape induced by $W_{\gamma}$ is of constant width $\pi / 2$.

The unit disc $D^{n+1}=\left\{x \in \mathbb{R}^{n+1} \mid\|x\| \leq 1\right\}$ of $\mathbb{R}^{n+1}$ is clearly self-dual. Let $R$ be a rotation of $\mathbb{R}^{n+2}$ about an $n$ dimensional linear subspace with a small angle. Then, since the property of constant width is an invariant property by $R$, by Theorem $11, I d^{-1} \circ \alpha_{N}\left(R\left(\alpha_{N}^{-1} \circ \operatorname{Id}\left(D^{n+1}\right)\right)\right)$ is selfdual as well (see Figure 17). Moreover, let $\widetilde{\triangle}$ be a spherical triangle of constant width $\pi / 2$ in $S^{2}$ containing $N$ as an interior point. Then, by Theorem 11, not only $I d^{-1} \circ \alpha_{N}(\widetilde{\triangle})$ itself, but also any $I d^{-1} \circ$ $\alpha_{N}(R(\widetilde{\triangle}))$ is self-dual (see Figure 18). For more consideration on simple, explicit examples, see [24].

On the other hand, any Reuleaux triangle in $\mathbb{R}^{2}$ containing the origin as an interior point (see Figure 19) is not a self-dual Wulff shape, although it is a Wulff shape of constant width in $\mathbb{R}^{2}$. This is because any Reuleaux triangle is strictly convex, and thus the boundary of it must be smooth by Theorem 8 if it is self-dual. However, there are three non-smooth points for any Reuleaux triangle in $\mathbb{R}^{2}$. By Theorem 11, its spherical convex body is not of constant width $\pi / 2$.

\subsection{Stability of $C^{\infty}$ convex integrands}

For the proof of the main theorem of this subsection (Theorem 13), the spherical method is not required. However, since the results in Subsection 3.8 may be regarded as the next step of Theorem 13 and they heavily depends on the spherical method, for the sake of readers' convenience, stability of $C^{\infty}$ convex integrands is quickly reviewed in this subsection. 

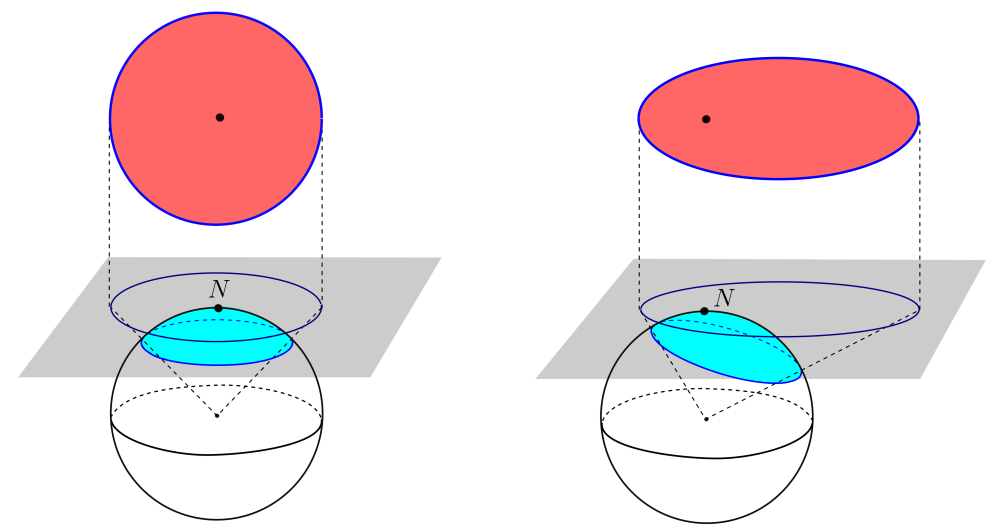

Fig. 17. Self-dual Wulff shapes include central projections of spherical caps of width $\pi / 2$.
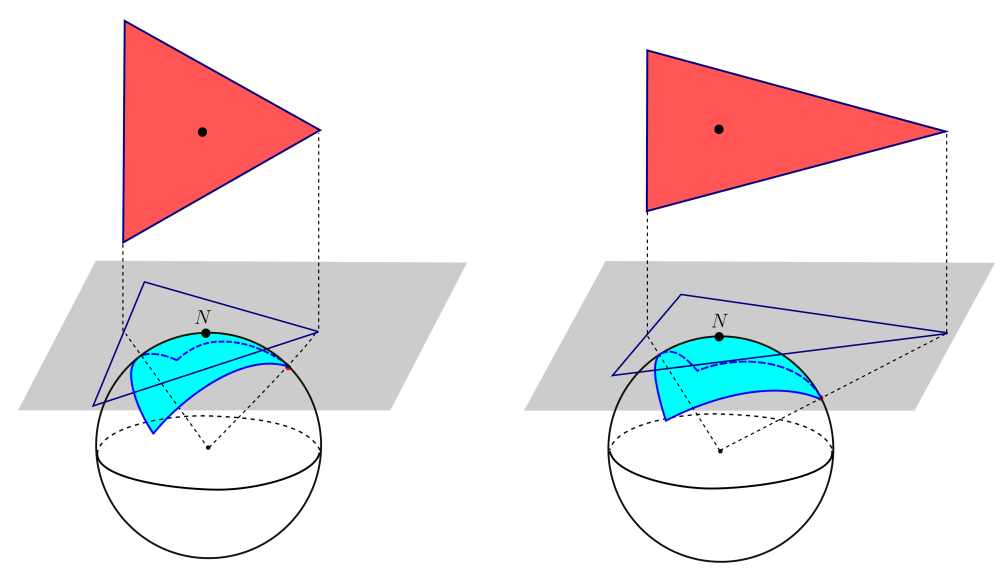

Fig. 18. Self-dual Wulff shapes include triangles which are central projections of constant-width spherical triangles of width $\pi / 2$. 


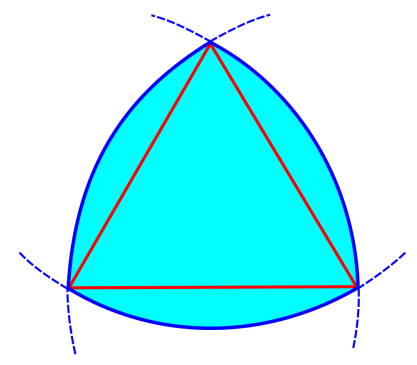

Fig. 19. Reuleaux triangle.

We start to review the definition of Whitney $C^{\infty}$ topology. Let $m, p$ be positive integers and let $M$ (resp., $P$ ) be a $C^{\infty}$ manifold of dimension $m$ (resp., dimension $p$ ). For any positive integer $r$, following [21], we define the $r$ jet bundle $J^{r}(M, P)$ as follows.

Definition 20 ([21]). Let $x$ be a point of $M$. Let $f, g: M \rightarrow P$ be $C^{\infty}$ mappings.

(1) $f$ has zero order contact with $g$ at $x$ if there exists a point $y \in P$ such that $f(x)=g(x)=y$.

(2) Suppose that $f$ has zero order contact with $g$ at $x$. Then, $f$ has first order contact with $g$ at $x$ if $(d f)_{x}=(d g)_{x}$ as mappings of $T_{x} M \rightarrow T_{y} P$. This is written as $f \sim_{0} g$ at $x$.

(3) $f$ has $r$-th order contact with $g$ at $x$ if $(d f): T M \rightarrow T P$ has $(r-1)$-th order contact with $g$ at every point of $T_{x} M$. This is written as $f \sim_{r} g$ at $x$.

(4) Let $J^{0}(M, P)_{x, y}$ denote the set consisting of equivalence classes under " $\sim_{0}$ at $x$ " of $C^{\infty}$ mappings $f: M \rightarrow P$.

(5) Let $J^{r}(M, P)_{x, y}$ denote the set consisting of equivalence classes under " $\sim_{r}$ at $x$ " of $C^{\infty}$ mappings $f: M \rightarrow P$ where $f(x)=y$.

(6) Let $J^{r}(M, P)=\bigcup_{(x, y) \in M \times P} J^{r}(M, P)_{x, y}$ (disjoint union). An element $\sigma$ in $J^{r}(M, P)$ is called a $r$-jet of mappings (or just a $r$-jet) from $M$ to $P$.

(7) Given a $C^{\infty}$ mapping $f: M \rightarrow P$, the mapping $j^{r}: M \rightarrow$ $J^{r}(M, P)$, called the $r$-jet extension of $f$, is defined by $j^{r} f(x)=$ equivalence class of $f$ in $J^{r}(M, P)_{x, f(x)}$.

Definition $21([21])$. (1) Denote by $C^{\infty}(M, P)$, the set consisting of $C^{\infty}$ mappings from $M$ to $P$. 
(2) Fix a non-negative integer $r$. Let $U$ be a subset of $J^{r}(M, P)$. Then, denote by $V(U)$ the set

$$
\left\{f \in C^{\infty}(M, P) \mid j^{r} f(M) \subset U\right\} .
$$

Notice that $V\left(U_{1}\right) \cap V\left(U_{2}\right)=V\left(U_{1} \cap U_{2}\right)$.

(3) The family of sets $\{V(U)\}$ form a basis for a topology on $C^{\infty}(M, P)$, where $U$ is an open subset of $J^{r}(M, P)$. This topology is called the Whitney $C^{r}$ topology. Denote by $W_{r}$ the set of open subsets of $C^{\infty}(M, P)$ in the Whitney $C^{r}$ topology.

(4) The Whitney $C^{\infty}$ topology on $C^{\infty}(M, P)$ is the topology whose basis is $W=\bigcup_{r=0}^{\infty} W_{r}$. This is a well-defined basis since $W_{r_{1}} \subset$ $W_{r_{2}}$ whenever $r_{1} \leq r_{2}$.

Definition $22([21])$. (1) Let $f, g$ be elements of $C^{\infty}(M, P)$. Then $f$ is said to be $\mathcal{A}$-equivalent to $g$ if there exist $C^{\infty}$ diffeomorphisms $h: M \rightarrow M$ and $H: P \rightarrow P$ such that the equality $f=H^{-1} \circ g \circ h$ holds.

(2) Let $f$ be in $C^{\infty}(M, P)$. Then, $f$ is said to be stable if there exists a neighborhood $W_{f}$ of $f$ in $C^{\infty}(M, P)$ such that each $g$ in $W_{f}$ is $\mathcal{A}$-equivalent to $f$.

(3) Denote the set consisting of stable mappings $f: M \rightarrow P$ by $S^{\infty}(M, P)$.

For more details on Jet bundles, the Whitney $C^{\infty}$ topology and stable mappings, for instance refer to $[5,21]$. By definition, it is clear that $S^{\infty}(M, P)$ is an open subset of $C^{\infty}(M, P)$.

Next, we review Mather's well-known answer to the structual stability problem posed by $\mathrm{R}$. Thom in [71].

Definition 23. Let $M, P$ be $C^{\infty}$ manifolds.

(1) A $C^{\infty}$ mapping $f: M \rightarrow P$ is said to be proper if $f^{-1}(C)$ is compact for any compact subset $C \subset P$.

(2) The subset of $C^{\infty}(M, P)$ consisting of proper mappings is denoted by $C_{\text {pro }}^{\infty}(M, P)$.

Question 12. Is the intersection $S^{\infty}(M, P) \cap C_{\text {pro }}^{\infty}(M, P)$ dense in $C_{\text {pro }}^{\infty}(M, P) ?$

Question 12 was completely solved by J. Mather ([45]). Mather's answer has the following surprizing form.

Theorem 12 ([45]). Suppose that $C_{\mathrm{pro}}^{\infty}(M, P)$ is not empty. Then, $S^{\infty}(M, P) \cap C_{\mathrm{pro}}^{\infty}(M, P)$ is dense in $C_{\mathrm{pro}}^{\infty}(M, P)$ if and only if the dimension-pair $(m, p)$ satisfies one of the following conditions. 
(1) $m<\frac{6}{7} p+\frac{8}{7}$ and $p-m \geq 4$

(2) $m<\frac{6}{7} p+\frac{9}{7}$ and $3 \geq p-m \geq 0$.

(3) $p<8$ and $p-m=-1$.

(4) $p<6$ and $p-m=-2$.

(5) $\quad p<7$ and $p-m \leq-3$.

Notice that $M=S^{n}$ and $P=\mathbb{R}_{+}$in our case. Thus, $(m, p)=(m, 1)$ in our case. In this case, one of the conditions (2)-(5) of Theorem 12 is satisfied. Moreover, since $S^{n}$ is compact, any $C^{\infty}$ function $\gamma: S^{n} \rightarrow \mathbb{R}_{+}$ is proper. Hence, we have the following.

Corollary 4 ([45]). Let $n$ be a positive integer. Then, the open subset $S^{\infty}\left(S^{n}, \mathbb{R}_{+}\right)$is dense in $C^{\infty}\left(S^{n}, \mathbb{R}_{+}\right)$.

For proper $C^{\infty}$ mappings of special type, it is natural to ask the similar question, namely to ask "Are generic proper mappings of special type stable ?". Such investigations, for instance, can be found in [46] for generic projections of submanifolds, in [12] for generic projections of stable mappings and in $[26,27,28,29,30]$ for generic distance-squared mappings and their generalizations. Motivated by these researches, we posed Question 9 in Section 1. We have a complete answer to Question 9 as follows.

Theorem $13([7])$. The open subset $S^{\infty}\left(S^{n}, \mathbb{R}_{+}\right) \cap C_{\mathrm{conv}}^{\infty}\left(S^{n}, \mathbb{R}_{+}\right)$ is dense in $C_{\mathrm{conv}}^{\infty}\left(S^{n}, \mathbb{R}_{+}\right)$.

In order to prove Theorem 13, it is better to replace an element of $S^{\infty}\left(S^{n}, \mathbb{R}_{+}\right)$with an easily treatable function. Thus, we want to have a geometric characterization of an element of $S^{\infty}\left(S^{n}, \mathbb{R}_{+}\right)$. Mather's celebrated geometric characterization of a proper stable mapping can be found in [44]. Notice that any $C^{0}$ function $S^{n} \rightarrow \mathbb{R}_{+}$is proper. Thus, it is easily seen that the following well-known geometric characterization of a stable function $S^{n} \rightarrow \mathbb{R}_{+}$is derived from the geometric characterization given in [44].

Proposition $15([44])$. Let $\gamma: S^{n} \rightarrow \mathbb{R}_{+}$be a $C^{\infty}$ function. Then, $\gamma$ is stable if and only if all critical points of $\gamma$ are non-degenerate and $\gamma\left(\theta_{1}\right) \neq \gamma\left(\theta_{2}\right)$ is satisfied for any two distinct critical points $\theta_{1}, \theta_{2} \in S^{n}$.

Notice that a proper stable function seems to be usually called a Morse function. However, a Morse function defined (for instance) in [21] or [49] is a $C^{\infty}$ function having only non-degenerate critical points, and thus it is a weaker notion than the notion of stable function in the sense of Mather. Therefore, in order to avoid unnecessary confusion, stable functions and Morse functions are distinguished in this survey article. 
Definition 24 ([49]). Let $\gamma: S^{n} \rightarrow \mathbb{R}_{+}$be a $C^{\infty}$ function and let $\theta \in S^{n}$ be a non-degenerate critical point of $\gamma$. Then, there exists a coordinate neighbourhood $(U, \varphi)$ of $\theta$ such that $\varphi(\theta)=\mathbf{0}$ and the following equality holds:

$$
\gamma \circ \varphi^{-1}\left(x_{1}, \ldots, x_{n}\right)=\gamma(\theta)-x_{1}^{2}-\cdots-x_{i}^{2}+x_{i+1}^{2}+\cdots+x_{n}^{2} .
$$

The integer $i(0 \leq i \leq n)$ does not depend on the particular choice of the coordinate neighbourhood $(U, \varphi)$ and it is called the Morse index of $\gamma$ at $\theta$.

\subsection{Simultaneous stability of $C^{\infty}$ convex integrands and their duals}

Let $\gamma: S^{n} \rightarrow \mathbb{R}_{+}$be a convex integrand. Recall that the dual convex integrand of $\gamma$ is $\delta: S^{n} \rightarrow \mathbb{R}_{+}$such that the following holds (see Section $1)$.

$$
\operatorname{inv}(\operatorname{graph}(\delta))=\partial \mathcal{W}_{\gamma}
$$

In this subsection, results on simultaneous stability of $\gamma$ and $\delta$ are summarized.

Define the functions $\widehat{\gamma}, \widehat{\delta}: S^{n} \rightarrow \mathbb{R}_{+}$by

$$
\widehat{\gamma}(\theta)=\frac{1}{\gamma(-\theta)} \text { and } \widehat{\delta}(\theta)=\frac{1}{\delta(-\theta)} \quad\left(\forall \theta \in S^{n}\right)
$$

respectively. By Proposition 8 of Subsection 2.5, we have the following.

$$
\partial \mathcal{D} \mathcal{W}_{\gamma}=\partial \mathcal{W}_{\delta}=\operatorname{inv}(\operatorname{graph}(\gamma))=\operatorname{graph}(\hat{\gamma}) .
$$

Notice that for any convex integrand $\gamma: S^{n} \rightarrow \mathbb{R}_{+}$, the Wulff shape $\mathcal{W}_{\gamma}$ is a convex body such that the origin is contained in it's interior. In Convex Body Theory, there is the notion of dual for a convex body containing the origin as an interior point. Namely, in Convex Body Theory, the boundary of the dual of $\mathcal{W}_{\gamma}$ is the following set (see for example [67]).

$$
\left\{\left(\theta, \frac{1}{\gamma(\theta)}\right) \mid \theta \in S^{n}\right\}
$$

However, the notion of dual in this sense seems to have less relations with the notion of pedal which is very important for this article and a common background in Physics (for instance, see [63]). On the other hand, the notion of dual Wulff shapes in our sense is closely related to the notion of pedal. Moreover, via the central projection, the pedal of $C^{\infty}$ embedding $\Phi: S^{n} \rightarrow \mathbb{R}^{n+1}-\{\mathbf{0}\}$ defined by $\Phi_{\delta}(\theta)=(\theta, \hat{\delta}(\theta))$ 
relative to the origin is characterized by using the spherical dual of the corresponding embedding $\widetilde{\Phi}: S^{n} \rightarrow S^{n+1}$; and the spherical dual is a well-known notion in Singularity Theory (for details, see Subsection 2.2 ). Since the notion of pedal is very important for our study, we adopt $\mathcal{D} \mathcal{W}_{\gamma}$ as the notion of dual Wulff shape of $\mathcal{W}_{\gamma}$.

For the simultaneous stability of $C^{\infty}$ convex integrands and their duals, we have the following:

Theorem $14([8])$. Let $\gamma: S^{n} \rightarrow \mathbb{R}_{+}$be a stable convex integrand and let $\delta$ be the dual convex integrand of $\gamma$. Then, $\delta$ is stable.

In order to prove Theorem 14, the following three assertions are needed.

Define the function $\widetilde{\gamma}: S^{n} \rightarrow \mathbb{R}_{+}$by $\widetilde{\gamma}(\theta)=d\left(\widetilde{\Psi}_{\widehat{\gamma}}(\theta), N\right)$.

Lemma $3.4([8])$. There exists a point $\theta$ of $S^{n}$ which is a degenerate critical point of $\widetilde{\gamma}$ if and only if the north pole $N$ is contained in the spherical caustic of $\widetilde{\Psi}_{\widehat{\gamma}}$.

For the proof of Lemma 3.4, we need the well-known fact that the singular set $S(\Phi)$ of the following mapping $\Phi$ is an $(n+1)$-dimensional $C^{\infty}$ submanifold of $S^{n} \times S_{N,+}^{n+1}$ (see for instance [64,66]).

$$
\begin{aligned}
\Phi: S^{n} \times S_{N,+}^{n+1} & \rightarrow \mathbb{R} \times S_{N,+}^{n+1} \\
(\theta, P) & \mapsto\left(d\left(\widetilde{\Psi}_{\hat{\gamma}}(\theta), P\right), P\right) .
\end{aligned}
$$

Lemma $3.5([8])$. The origin $\mathbf{0}$ is a point of symmetry set of $\partial \mathcal{W}$ if and only if the north polar $N$ is a point of spherical symmetry set of $\partial \widetilde{\mathcal{W}}$.

Remark 3.1. Notice that $\gamma(\theta)=\tan (\widetilde{\gamma}(\theta))$ for any $\theta \in S^{n}$. Since the function $\tan :(0, \pi / 2) \rightarrow \mathbb{R}_{+}$is a $C^{\infty}$ diffeomorphism, it follows that $\theta$ is a non-degenerate critical point of $\gamma$ if and only if $\theta$ is a nondegenerate critical point of $\widetilde{\gamma}$.

Proposition 16 ([8]). Let $\mathcal{W}_{\gamma}$ be the Wulff shape associated with $\gamma$ and let $\widetilde{\mathcal{W}}_{\gamma}$ be the spherical Wulff shape associated with $\mathcal{W}_{\gamma}$. Then the following holds:

(1) Spherical-Caust $\left(\partial \widetilde{\mathcal{W}}_{\gamma}\right)=$ Spherical-Caust $\left(\partial \mathcal{D} \widetilde{\mathcal{W}}_{\gamma}\right)$.

(2) Spherical-Sym $\left(\partial \widetilde{\mathcal{W}}_{\gamma}\right)=$ Spherical-Sym $\left(\partial \mathcal{D} \widetilde{\mathcal{W}}_{\gamma}\right)$.

Proposition 16 is one of merits of the spherical method because the corresponding Euclidean properties do not hold in general.

By Theorem 14, we have the following corollary. 
Corollary 5 ([8]). Let $\gamma: S^{n} \rightarrow \mathbb{R}_{+}$be a convex integrand and let $\delta: S^{n} \rightarrow \mathbb{R}_{+}$be the dual convex integrand of $\gamma$. Then, the following are equivalent.

(1) The convex integrand $\gamma$ is stable.

(2) The convex integrand $\delta$ is stable.

(3) The function $\widehat{\gamma}$, whose graph is exactly $\partial \mathcal{W}_{\delta}=\partial \mathcal{D} \mathcal{W}_{\gamma}$, is stable.

(4) The function $\widehat{\delta}$, whose graph is exactly $\partial \mathcal{W}_{\gamma}=\partial \mathcal{D} \mathcal{W}_{\delta}$, is stable.

Next, more detailed dual relationships for stable convex integrands $\gamma, \delta$ are explained.

Theorem $15([8])$. Let $\gamma: S^{n} \rightarrow \mathbb{R}_{+}$be a stable convex integrand and let $\delta: S^{n} \rightarrow \mathbb{R}_{+}$be the dual convex integrand of $\gamma$. Then, the following hold:

(1) $\quad A$ point $\theta_{0} \in S^{n}$ is a non-degenerate critical point of $\gamma$ if and only if $-\theta_{0} \in S^{n}$ is a non-degenerate critical point of $\delta$.

(2) Suppose that $\theta_{0} \in S^{n}$ is a non-degenerate critical point of $\gamma$. Then, for any $i(0 \leq i \leq n)$, the Morse index of $\gamma$ at $\theta_{0}$ is $i$ if and only if the Morse index of $\delta$ at $-\theta_{0}$ is $(n-i)$.

For the proof of Theorem 15, not only the spherical method, but also Andrews formulas ([1]), which is explained quickly below, is needed.

Let $\gamma: S^{n} \rightarrow \mathbb{R}_{+}$be a $C^{\infty}$ convex integrand and let $\delta: S^{n} \rightarrow \mathbb{R}_{+}$ be the dual convex integrand of $\gamma$. Notice that $\partial \mathcal{W}_{\gamma}$ (resp., $\left.\partial \mathcal{D} \mathcal{W}_{\gamma}\right)$ is the image of the embedding $\Phi_{\delta}$ (resp., $\Phi_{\gamma}$ ) defined by $\Phi_{\delta}(\theta)=(\theta, \widehat{\delta}(\theta))$ (resp., $\Phi_{\gamma}(\theta)=(\theta, \widehat{\gamma}(\theta))$ ). Define the mapping $h_{\delta}: S^{n} \rightarrow S^{n}$ (resp., $h_{\gamma}: S^{n} \rightarrow S^{n}$ ) so that $\gamma(\theta)$ (resp., $\delta(\theta)$ ) is the perpendicular distance from the origin 0 to the affine tangent hyperplane to $\Phi_{\delta}\left(S^{n}\right)$ (resp., $\left.\Phi_{\gamma}\left(S^{n}\right)\right)$ at $\Phi_{\delta}\left(h_{\delta}(\theta)\right)$ (resp., $\left.\Phi_{\gamma}\left(h_{\gamma}(\theta)\right)\right)$. Then, the following holds.

Proposition $17([8]) . \quad$ (1) Both of $h_{\delta}$ and $h_{\gamma}$ are $C^{\infty}$ diffeomorphisms.

(2) Both of $\Phi_{\delta}\left(S^{n}\right)$ and $\Phi_{\gamma}\left(S^{n}\right)$ are strictly locally convex.

Therefore, by Andrews [1], the following equalities hold for any $\theta \in S^{n}$ (see Figure 20 where $T_{1}$ (resp., $T_{2}$ ) denotes the affine tangent hyperplane to $\Phi_{\delta}\left(S^{n}\right)$ (resp., $\left.\Phi_{\gamma}\left(S^{n}\right)\right)$ at $\Phi_{\delta}\left(h_{\delta}(\theta)\right)$ (resp., $\left.\Phi_{\gamma}\left(h_{\gamma}(\theta)\right)\right)$ ). These two equalities are called Andrews's formulas.

$$
\begin{aligned}
\Phi_{\delta}\left(h_{\delta}(\theta)\right) & =\gamma(\theta) \theta+\nabla \gamma(\theta) \\
\Phi_{\gamma}\left(h_{\gamma}(\theta)\right) & =\delta(\theta) \theta+\nabla \delta(\theta) .
\end{aligned}
$$


Here, $\nabla \gamma(\theta)$ (resp., $\nabla \delta(\theta)$ ) stands for the gradient vector of $\gamma$ (resp., $\delta$ ) at $\theta \in S^{n}$ with respect to the standard metric on $S^{n}$.
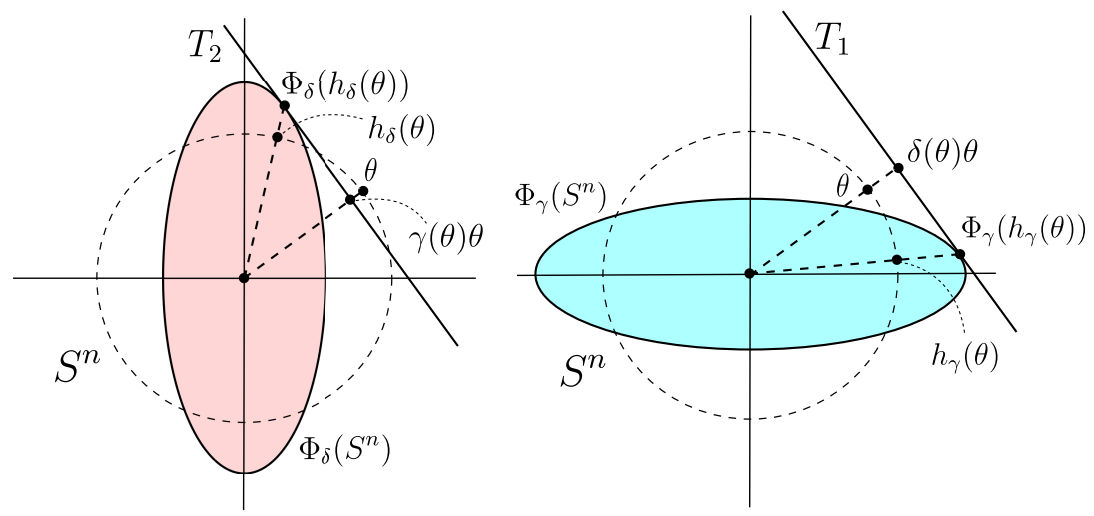

Fig. 20. Andrews's formulas

It is clear that, by Theorem 15, we have the following corollary.

Corollary 6 ([8]). Let $\gamma: S^{n} \rightarrow \mathbb{R}_{+}$be a stable convex integrand and let $\delta: S^{n} \rightarrow \mathbb{R}_{+}$be the dual convex integrand of $\gamma$. Moreover, let $\theta_{0}$ be a point of $S^{n}$ and let $i$ be an integer such that $0 \leq i \leq n$. Then, the following are equivalent.

(1) The point $\theta_{0} \in S^{n}$ is a non-degenerate critical point of $\gamma$ with Morse index $i$.

(2) The point $-\theta_{0} \in S^{n}$ is a non-degenerate critical point of $\delta$ with Morse index $(n-i)$.

(3) The point $-\theta_{0} \in S^{n}$ is a non-degenerate critical point of $\hat{\gamma}$ with Morse index $(n-i)$.

(4) The point $\theta_{0} \in S^{n}$ is a non-degenerate critical point of $\widehat{\delta}$ with Morse index $i$.

\section{$\S 4$. Questions}

In this section, we pose questions related to the topics in Section 3. To the best of authors' knowledge, all questions posed in this section except for Questions 14, 15 seem to be open. 


\subsection{On aperture of higher dimensional hypersurfaces}

Question 13. Let $M$ be a compact $C^{\infty}$ manifold of dimension $n$. Then, is it possible to obtain similar results as Theorems $3-5$ for a $C^{\infty}$ immersion $\mathbf{r}: M \rightarrow \mathbb{R}^{n+1}$ with $n \geq 2$ ?

In our setting, at the initial stage $(=$ before rotating all tangent spaces), the no-silhouette of $\mathbf{r}$ must exists, namely the set

$$
\mathcal{N} \mathcal{S}_{\mathbf{r}}=\mathbb{R}^{n+1}-\bigcup_{s \in M}\left(\mathbf{r}(s)+d \mathbf{r}_{s}\left(T_{s} M\right)\right)
$$

must be non-empty.

Proposition 18 ([59]). Let $M$ be a closed $C^{\infty}$ manifold of dimension $n$, and let $\mathbf{r}: M \rightarrow \mathbb{R}^{n+1}$ be a $C^{\infty}$ immersion with its no-silhouette. Suppose that $n \geq 2$. Then, the immersion $\mathbf{r}$ must be an embedding and $M$ must be $C^{\infty}$ diffeomorphic to $S^{n}$.

Firstly, we examine the case $n=2$. By this proposition, our initial situation is as follows:

Given a $C^{\infty}$ embedding $S^{2} \rightarrow \mathbb{R}^{3}$ such that

$$
\mathcal{N} \mathcal{S}_{\mathbf{r}}=\mathbb{R}^{3}-\bigcup_{s \in S^{2}}\left(\mathbf{r}(s)+d \mathbf{r}_{s}\left(T_{s}\left(S^{2}\right)\right)\right) \neq \emptyset .
$$

Next, from the initial situation, we would like to rotate all tangent planes about their rotation axes simultaneously with the same angle. So, the following question arises:

Question 14. Is it possible to choose rotation axes depending on $s \in S^{2}$ smoothly?

The answer to this question is "NO" by the following well-known result due to $\mathrm{H}$. Poincaré.

Proposition 19 (H. Poincaré). There is no $C^{\infty}$ non-zero vector field on $S^{2}$.

As for Poincaré's result, for instance refer to [50]. Secondly, we consider the case $n \geq 3$. By Proposition 18, our initial situation is as follows: "Given a $C^{\infty}$ embedding $S^{n} \rightarrow \mathbb{R}^{n+1}$ such that

$$
\mathcal{N} \mathcal{S}_{\mathbf{r}}=\mathbb{R}^{n+1}-\bigcup_{s \in S^{n}}\left(\mathbf{r}(s)+d \mathbf{r}_{s}\left(T_{s}\left(S^{n}\right)\right)\right) \neq \emptyset
$$

where $n \geq 3$."

Next, from the initial situation, we would like to rotate all tangent spaces ( $n$-dimensional vector spaces $d \mathbf{r}_{s}\left(T_{s}\left(S^{n}\right)\right)$ ) about their rotation 
axes $((n-1)$-dimensional vector spaces) simultaneously with the same angle. Then, we may ask the same question as in the case $n=2$.

Question 15. Is it possible to choose rotation axes depending on $s \in S^{n}$ smoothly?

Fortunately, by the celebrated result due to W. P. Thurston (Theorem 16), we already have the complete answer to Question 15.

Theorem $16([72])$. Let $M$ be a compact $C^{\infty}$ manifold of dimension $n$. Then, the following hold.

(1) The given manifold $M$ has a $C^{\infty}$ codimension one foliation if and only if $\chi(M)=0$, where $\chi(M)$ denotes the Euler characteristic of $M$.

(2) Every $(n-1)$-plane field $\tau^{n-1}$ on the given $M$ is homotopic to the tangent plane field of a $C^{\infty}$ codimension one foliation.

Corollary 7. It is possible to choose rotation axes depending on $s \in S^{n}$ smoothly if and only if $n$ is odd.

Let $\mathbf{r}: S^{2 k+1} \rightarrow \mathbb{R}^{2 k+2}$ be a $C^{\infty}$ embedding such that $\mathcal{N} \mathcal{S}_{\mathbf{r}} \neq \emptyset$. Moreover, we let $\mathcal{F}$ be a $C^{\infty}$ codimension one foliation of $S^{2 k+1}$. Then, the following rotated no-silhouette is well-defined:

$$
\mathcal{N} \mathcal{S}_{\theta, \mathbf{r}, \mathcal{F}}=\mathbb{R}^{2 k+2}-\bigcup_{s \in S^{2 k+1}}\left(\mathbf{r}(s)+R_{\theta, L_{s}} d \mathbf{r}_{s}\left(T_{s}\left(S^{2 k+1}\right)\right)\right),
$$

where $L_{s}$ is the leaf of $\mathcal{F}$ containing $s \in S^{2 k+1}$ and

$$
R_{\theta, L_{x}}: \mathbb{R}^{2 k+2} / d \mathbf{r}_{s}\left(T_{s}\left(L_{s}\right)\right) \rightarrow \mathbb{R}^{2 k+2} / d \mathbf{r}_{s}\left(T_{s}\left(L_{s}\right)\right)
$$

is the rotation defined by $R_{\theta, L_{x}}([x])=[x]\left(\begin{array}{rr}\cos \theta & \sin \theta \\ -\sin \theta & \cos \theta\end{array}\right)$. Thus, we may ask

Question 16. How is $\mathcal{N} \mathcal{S}_{\theta, \mathbf{r}, \mathcal{W}}$ growing as $\theta$ increases ?

Some of results in the plane curve case works as well. For instance, we have the following:

Lemma 4.1. Let $\mathbf{r}: S^{2 k+1} \rightarrow \mathbb{R}^{2 k+2}$ (resp., $\mathcal{F}$ ) be a $C^{\infty}$ embedding (resp., $C^{\infty}$ codimension one foliation of $S^{2 k+1}$ ). Then, $\mathcal{N S}_{\frac{\pi}{2}, \mathbf{r}, \mathcal{F}}$ is the empty set.

However, there are still many open questions. For instance,

Question 17. Is the aperture point well-defined in higher dimensional case? 
Question 18. Apart from no-silhouettes, investigate "rotated envelopes". Namely, for a given compact $C^{\infty}$ manifold $M$ of dimension $n$ satisfying $\chi(M)=0$, a given $C^{\infty}$ codimension one foliation $\mathcal{F}$ of $M$, a given $C^{\infty}$ immersion $\mathbf{r}: M \rightarrow \mathbb{R}^{n+1}$ and a given angle $\theta \in \mathbb{R}$, investigate the envelope of the family of $n$-planes in $\mathbb{R}^{n+1}$ :

$$
\left\{\mathbf{r}(s)+R_{\theta, L_{s}} d \mathbf{r}_{s}\left(T_{s} M\right)\right\}_{s \in M}
$$

where $L_{s}$ is the leaf of $\mathcal{F}$ containing $s \in M$.

\subsection{On the space consisting of convex integrands and the space consisting of Wulff shapes}

Recall again that the mapping

$$
\mathcal{W}: C^{0}\left(S^{n}, \mathbb{R}_{+}\right) \rightarrow \mathcal{H}_{\text {conv }, 0}\left(\mathbb{R}^{n+1}\right)
$$

defined by $\mathcal{W}(\gamma)=\mathcal{W}_{\gamma}$ has been introduced and properties of $\mathcal{W}$ has been studied in Section 1. In more details, it has been confirmed that the restriction of $\mathcal{W}$ to $C I\left(S^{n}, \mathbb{R}_{+}\right)$

$$
\left.\mathcal{W}\right|_{C I\left(S^{n}, \mathbb{R}_{+}\right)}: C I\left(S^{n}, \mathbb{R}_{+}\right) \rightarrow \mathcal{H}_{\text {conv }, \mathbf{0}}\left(\mathbb{R}^{n+1}\right)
$$

is bijective, where $C I\left(S^{n}, \mathbb{R}_{+}\right)$is the set consisting of convex integrands as defined in Subsection 3.5.

$$
C I\left(S^{n}, \mathbb{R}_{+}\right)=\left\{\gamma \in C^{0}\left(S^{n}, \mathbb{R}_{+}\right) \mid \gamma: \text { Convex Integrand }\right\}
$$

Moreover, it is not difficult to see that the restriction of $\mathcal{W}$ to $C I\left(S^{n}, \mathbb{R}_{+}\right)$ is continuous. We would like to study more detailed properties of the restriction of $\mathcal{W}$ to $C I\left(S^{n}, \mathbb{R}_{+}\right)$. Thus, we ask the following.

Question 19. Is the following mapping bi-Lipschitz?

$$
\left.\mathcal{W}\right|_{C I\left(S^{n}, \mathbb{R}_{+}\right)}: C I\left(S^{n}, \mathbb{R}_{+}\right) \rightarrow \mathcal{H}_{\text {conv }, \mathbf{0}}\left(\mathbb{R}^{n+1}\right) .
$$

Question 19 asks whether or not the two spaces $C I\left(S^{n}, \mathbb{R}_{+}\right)$and $\mathcal{H}_{\text {conv }, \mathbf{0}}\left(\mathbb{R}^{n+1}\right)$ cannot be distinguished in the sense of Lipschitz geometry. Notice that $C I\left(S^{n}, \mathbb{R}_{+}\right)$is the space consisting of convex integrands $S^{n} \rightarrow \mathbb{R}_{+}$, whereas the space $\mathcal{H}_{\text {conv }, \mathbf{0}}\left(\mathbb{R}^{n+1}\right)$ is a space consisting of some compact sets in $\mathbb{R}^{n+1}$. Therefore, the metrics of these two spaces are completely different. Roughly speaking, the metric of $C I\left(S^{n}, \mathbb{R}_{+}\right)$measures the difference of two functions $\gamma_{1}, \gamma_{2}: S^{n} \rightarrow \mathbb{R}_{+}$ only by the difference from radial directions. On the other hand, the metric of $\mathcal{H}_{\text {conv }, \mathbf{0}}\left(\mathbb{R}^{n+1}\right)$ measures the difference of two convex bodies $W_{1}, W_{2} \subset \mathbb{R}^{n+1}$ containing the origin as an interior point by the 
difference from all directions. Therefore, these two metrics are quite different. Nevertheless, to our surprise, there seems to be several signs which indicates that these two spaces might be bi-Lipschitz. ${ }^{2}$

\subsection{On convex integrands of class $C^{k}(2 \leq k<\infty)$}

Question 20. Let $\gamma: S^{n} \rightarrow \mathbb{R}_{+}$be a convex integrand and let $\delta: S^{n} \rightarrow \mathbb{R}_{+}$be the dual convex integrand of $\gamma$. Moreover, let $k$ be a positive integer greater than or equal to 2 . Then, are the following equivalent?

(1) The convex integrand $\gamma$ is of class $C^{k}$.

(2) The convex integrand $\delta$ is of class $C^{k}$.

(3) The function $\widehat{\gamma}$, whose graph is exactly $\partial \mathcal{W}_{\delta}=\partial \mathcal{D} \mathcal{W}_{\gamma}$, is of class $C^{k}$.

(4) The function $\widehat{\delta}$, whose graph is exactly $\partial \mathcal{W}_{\gamma}=\partial \mathcal{D} \mathcal{W}_{\delta}$, is of class $C^{k}$.

Notice that, as Figure 4 in Section 1 shows, it is impossible to replace $C^{k}$ class $(2 \leq k<\infty)$ with $C^{1}$ class.

The background of Question 20 is as follows. Historically, F. Morgan in [51] seems to be the first mathematician who treated such a kind of question although his result requires a mild assumption and the conclusion of his result is weak. Few years later after [51], H. M. Soner gave the same conclusion as Question 20 in [68] under a mild assumption on $\gamma$ (see also [20] where Soner's lemma (Lemma 1.7.2 in [20]) plays an important role for studying Wulff shapes by the level-set method). As for the $C^{\infty}$ class, under some mild assumptions on $\gamma$, not only F. Morgan ([51]) and H. M. Soner ([68]) but also B. Andrews ([1]) obtains the same conclusion as Question 20.

Moreover, if we succeed to solve Question 20 affirmatively, then we can simultaneously solve affirmative following question completely.

Question 21. Obtain the lowest $k$ so that $\gamma$ is of class $C^{k}$ if and only if $\partial \mathcal{W}_{\gamma}$ is a $C^{k}$ submanifold. ${ }^{3}$

\footnotetext{
${ }^{2}$ Note 1 added in revision. After a few months from the first submission, Question 19 was solved affirmatively in [25]. It is proved in [25] that the restriction of $\mathcal{W}$ to $C I\left(S^{n}, \mathbb{R}_{+}\right)$is an isometry. Thus, Question 19 has been solved in the strongest form.

${ }^{3}$ Note 2 added in revision. The authors learned from the referee that Andrews's formulas given in Subsection 3.8 works well if $\gamma: S^{n} \rightarrow \mathbb{R}_{+}$is convex and of class $C^{k}(k \geq 2)$ (cf. [62]). Therefore, in this case, $\partial \mathcal{W}_{\gamma}$ is of class $C^{k-1}$.
} 


\section{$\S$ Acknowledgements}

The authors would like to express their sincere gratitude to the anonymous referee for careful reading of this article and making invaluable suggestions.

\section{References}

[1] B. Andrews, Harnack inequalities for evolving hypersurfaces, Math. Z., 217 (1994), no. 2, 179-197.

[2] V.I. Arnol'd, Singularities of caustics and wave fronts, Kluwer Academic Publishers Group, Dordrecht, 1990.

[3] V. I. Arnol'd, The geometry of spherical curves and the algebra of quaternions, Russian Math. Surveys, 50 (1995), 1-68.

[4] V. I. Arnol'd, V. V. Goryunov, O. V. Lyashko and V. A. Vasil'ev, Dynamical Systems VIII, Encyclopaedia of Mathematical Sciences, 39, SpringerVerlag, Berlin Heidelberg New York, 1989.

[5] V.I. Arnol'd, S.M. Gusein-Zade and A.N. Varchenko, Singularities of Differentiable Maps I, Monographs in Mathematics, 82, Birkhäuser, Boston Basel Stuttgart, 1985.

[6] M. Barnsley, Fractals Everywhere 2nd edition, Morgan Kaufmann Pub., San Fransisco, 1993

[7] E. B. Batista, H. Han and T. Nishimura, Stability of $C^{\infty}$ convex integrands, to appear in Kyushu J. Math. (A preprint version is available from arXiv:1601.06347 [math.GT]).

[ 8 ] E. B. Batista, H. Han and T. Nishimura, Simultaneous stability of $C^{\infty}$ convex integrands and their duals, preprint (available from arXiv:1603.08331 [math.GT]).

[9] J.W. Bruce and P.J. Giblin, Growth, motion and 1-parameter families of symmetry sets, Proc. Roy. Soc. Edinburgh Sect. A, 104 (1986), 179-204.

[10] J.W. Bruce and P.J. Giblin, Curves and Singularities (second edition), Cambridge University Press, Cambridge, 1992.

[11] J.W. Bruce, P.J. Giblin and C.G. Gibson, Symmetry sets, Proc. Roy. Soc. Edinburgh Sect. A, 101(12) (1985), 163-186.

[12] J.W. Bruce and N.P. Kirk, Generic projections of stable mappings, Bull. London Math. Soc., 32 (2000), 718-728.

[13] B. Dacorogna and C.E. Pfister, Wulff theorem and best constant in Sobolev inequality, J. Math. Pures Appl., 71(1992), 97-118.

[14] A. Dinghas, Uber einen geometrischen Satz von Wulff f'ur die Gleichgewichts form von Kristallen, Z. Kristallogr., Mineral. Petrogr., 105 (1944), 304-314.

[15] K. Falconer, Fractal Geometry-Mathematical Foundations and applications 2nd edition, John Wiley \& Sons Ltd., Chichester, West Sussex, 2003. 
[16] I. Fonseca, The Wulff theorem revisited, Proc. Roy. Soc. London Ser. A, 432 (1991), 125-145.

[17] I. Fonseca and S. Müller, A uniqueness proof for the Wulff theorem, Proc. Roy. Soc. Edinburgh Sect. A, 119 (1991), 125-136.

[18] F. C. Frank, Metal Surfaces, ASM, Cleveland, OH, 1963.

[19] S. Gao, D. Hug and R. Schneider, Intrinsic volumes and polar sets in spherical space, Math. Notae, 41 (2001/02), 159-176(2003).

[20] Y. Giga, Surface Evolution Equations, Monographs of Mathematics, 99, Springer, 2006.

[21] M. Golubitsky and V. Guillemin, Stable mappings and their singularities, Graduate Texts in Mathematics 14, Springer, New York, 1973.

[22] H. Han and T. Nishimura, The spherical dual transform is an isometry for spherical Wulff shapes, to appear in Studia Math. (A preprint version is available from arXiv:1504.02845 [math.MG]).

[23] H. Han and T. Nishimura, Strictly convex Wulff shapes and $C^{1}$ convex integrands, to appear in Proc. Amer. Math. Soc. (A preprint version is available from arXiv:1507.05162 [math.MG]).

[24] H. Han and T. Nishimura, Self-dual Wulff shapes and spherical convex bodies of constant width $\pi / 2$, to appear in J. Math. Soc. Japan (A preprint version is available from arXiv:1511.04165 [math.MG]).

[25] H. Han and T. Nishimura, The Wulff construction for convex integrands, preprint (available from arXiv:1607.02885 [math.MG]).

[26] S. Ichiki and T. Nishimura, Distance-squared mappings, Topology Appl., 160 (2013), 1005-1016.

[27] S. Ichiki and T. Nishimura, Recognizable classification of Lorentzian distance-squared mappings, J. Geom. Phys., 81 (2014), 62-71.

[28] S. Ichiki and T. Nishimura, Around distance-squared mappings, RIMS Kôkyûroku, 1948 (2015), 28-37.

[29] S. Ichiki and T. Nishimura, Generalized distance-squared mappings of $\mathbb{R}^{n+1}$ into $\mathbb{R}^{2 n+1}$, Real and complex singularities, 121-132, Contemp. Math., 675, Amer. Math. Soc., Providence, RI, 2016.

[30] S. Ichiki, T. Nishimura, R. Oset Sinha and M.A.S. Ruas, Generalized distance-squared mappings of the plane into the plane, Adv. Geom., 16 (2016), 189-198.

[31] S. Izumiya, Differential geometry from the viewpoint of Lagrangian or Legendrian singularity theory, in Singularity Theory, Proceedings of the 2005 Marseille Singularity School and Conference, ed., D. Chéniót et al., World Scientic (2007), 241-275.

[32] S. Izumiya and G. Ishikawa, Applied Singularity Theory, Kyoritsu Syuppan Co., LTD, 1998 (in Japanese).

[33] S. Izumiya and M. Takahashi, Caustics and wave front propagations: Applications to differential geometry, Banach Center Publ., 82 (2008), 125-142.

[34] S. Izumiya and F. Tari, Projections of hypersurfaces in the hyperbolic space to hyperhorospheres and hyperplanes, Rev. Mat. Iberoam., 24 (2008), 895-920. 
[35] D. Kagatsume and T. Nishimura, Aperture of plane curves, J. Singul., 12 (2015), 80-91.

[36] K. Kitagawa and T. Nishimura, Classification of singularities of pedal curves in $S^{2}$, The Natural Sciences, Journal of the Faculty of Education and Human Sciences, Yokohama National University, 10 (2008), 39-55.

[37] M. Lassak, Width of spherical convex bodies, Aequationes Math., 89 (2015), $555-567$.

[38] M. Lassak, Reduced spherical polygons, Colloq. Math., 138 (2015), 205-216.

[39] H. Maehara, Geometry of Circles and Spheres, Asakura Publishing, 1998 (in Japanese).

[40] J.N. Mather, Stability of $C^{\infty}$ mappings, I. The division theorem, Ann. of Math., 87 (1968), 89-104.

[41] J.N. Mather, Stability of $C^{\infty}$ mappings, II. Infinitesimal stability implies stability, Ann. of Math., 89 (1969), 254-291.

[42] J.N. Mather, Stability of $C^{\infty}$ mappings, III. Finitely determined mapgerms, Publ. Math. Inst. Hautes Études Sci., 35 (1969), 127-156.

[43] J.N. Mather, Stability of $C^{\infty}$ mappings, IV. Classification of stable mapgerms by $\mathbb{R}$-algebras, Publ. Math. Inst. Hautes Études Sci., 37 (1970), 223-248.

[44] J.N. Mather, Stability of $C^{\infty}$-mappings V. Transversality, Adv. in Math., 4 (1970), 301-336.

[45] J.N. Mather, Stability of $C^{\infty}$-mappings VI. The nice dimensions, Lecture Notes in Math., 192 (1971), 207-253.

[46] J.N. Mather, Generic projections, Ann. of Math., 98 (1973), 226-245.

[47] J. Matousek, Lectures on Discrete Geometry, Springer, 2002.

[48] J. L. Meijering, Usefulness of a $1 / \gamma$ plot in the theory of thermal etching, Acta Metallurgica, 11 (1963), 847-849.

[49] J. W. Milnor, Morse Theory, Annals of Mathematics Studies 51, Princeton University Press, Princeton, New Jersey, 1963.

[50] J. W. Milnor, Topology from the differentiable viewpoint. Revised version. Princeton Landmarks in Mathematics, Princeton University Press, Princeton, New Jersey, 1997.

[51] F. Morgan, The cone over the Clifford torus in $\mathbb{R}^{4}$ is F-minimizing, Math. Ann., 289 (1991), 341-354.

[52] F. Morgan, Private communications, 2015.

[53] T. Nishimura, Singularities of tangent pedal curves in $S^{3}$, The Natural Sciences, Journal of the Faculty of Education and Human Sciences, Yokohama National University, 9 (2007), 43-50.

[54] T. Nishimura, Normal forms for singularities of pedal curves produced by non-singular dual curve germs in $S^{n}$, Geom Dedicata, 133 (2008), 59-66.

[55] T. Nishimura, Singularities of pedal curves produced by singular dual curve germs in $S^{n}$, Demonstratio Math., 43 (2010), 447-459.

[56] T. Nishimura, Singularities of one-parameter pedal unfoldings of spherical pedal curves, J. Singul., 2 (2010), 160-169. 
[57] T. Nishimura, Whitney umbrellas and swallowtails, Pacific J. Math., 252 (2011), 459-471.

[58] T. Nishimura, Wave front evolution and pedal evolution, RIMS Kôkyûroku Bessatsu, B38 (2013), 15-30.

[59] T. Nishimura and Y. Sakemi, View from inside, Hokkaido Math. J., 40 (2011), 361-373.

[60] T. Nishimura and Y. Sakemi, Topological aspect of Wulff shapes, J. Math. Soc. Japan, 66 (2014), 89-109.

[61] A. Ookawa, Crystal growth. Reprinted Edition, Kyoritsu Shuppan Co., Ltd., 2001 (in Japanese).

[62] B. Palmer, Stability of the Wulff shape, Proc. Amer. Math Soc., 126 (1998), 3661-3667.

[63] A. Pimpinelli and J. Villain, Physics of Crystal Growth, Monographs and Texts in Statistical Physics, Cambridge University Press, Cambridge New York, 1998.

[64] I. R. Porteous, The normal singularities of a submanifold, J. Diff. Geom., 5 (1971), 543-564.

[65] S. A. Robertson and M. C. Romero-Fuster, The convex hull of a hypersurface, Proc. London Math. Soc., 50 (1985), 370-384.

[66] M. C. Romero-Fuster and M. A. S. Ruas, Some stability questions concerning caustics for different propagation laws, Portugal. Math., 51 (1994), 595-605.

[67] R. Schneider, Convex Bodies: The Brunn-Minkowski Theory 2nd Edition, Encyclopedia of Mathematics and its Applications 44, Cambridge University Press, Cambridge, 2013.

[68] H. M. Soner, Motion of a set by the curvature of its boundary, J. Differential Equations, 101 (1993), 313-372.

[69] J. E. Taylor, Crystalline variational problems, Bull. Amer. Math. Soc., 84 (1978), 568-588.

[70] J. E. Taylor, J. W. Cahn and C. A. Handwerker, Geometric models of crystal growth, Acta Metallurgica et Materialia, 40 (1992), 1443-1474.

[71] R. Thom and H. Levine, Singularities of differentiable mappings, Springer Lecture Notes in Mathematics, 192, Springer-Verlag, Berlin, 1-89 (1971).

[72] W. P. Thurston, Existence of codimension-one foliations, Ann. of Math., 104 (1976), 249-268.

[73] G. Wulff, Zur frage der geschwindindigkeit des wachstrums und der auflösung der krystallflachen, Z. Kristallographine und Mineralogie, 34 (1901), 449-530.

[74] V. M. Zakalyukin, Singularities of convex hulls of smooth maniifolds, Functional Anal. Appl., 11 (1977), 225-227(1978). 
Graduate School of Environment and Information Sciences, Yokohama National University, Yokohama 240-8501, Japan

E-mail address: han-huhe-bx@ynu.jp

Research Institute of Environment and Information Sciences, Yokohama National University, Yokohama 240-8501, Japan

E-mail address: nishimura-takashi-yx@ynu.jp 